Part of this work has been performed in the University of Southern California. The authors are indebted to the Department of Mathematics of USC and in particular Professor W.-A. Harris, Jr, for their hospitality and support. After the revision of this paper we learned that Professor Harris passed away. We express our deep feelings of sorrow at his decease. 
Pacific

Journal of

Mathematics

THE STOKES PHENOMENON IN EXACT ASYMPTOTICS

B.L.J. Braaksma, G.K. Immink, and Y. Sibuya 


\title{
THE STOKES PHENOMENON IN EXACT ASYMPTOTICS
}

\author{
B.L.J. BraAksma, G.K. Immink, and Y. Sibuya \\ Dedicated to Prof. H.L. Turrittin on the occasion of his ninetieth birthday
}

\begin{abstract}
As an introduction we present a new, elementary and constructive proof of the multisummability properties of formal solutions of linear ODE's at irregular singular points. This serves to illustrate the geometric approach to multisummation. Basic properties of multisums and the associated sheaves are derived. Next, we study Cauchy-Heine transforms in relation to multisummation and the Stokes phenomenon. We show how to construct multisums with a prescribed Stokes phenomenon, using the Malgrange-Sibuya isomorphism. Starting from the Stokes automorphisms we introduce the alien derivations of J. Ecalle and derive Ecalle's bridge equation for the general integral of linear ODE's. The main ideas are illustrated with some very simple examples.
\end{abstract}

\section{Introduction.}

Consider the differential equation $(D): D y=0$, where $D$ is a linear differential operator of order $n$, with coefficients in $\mathbf{C}\{z\}$. It has $n$ independent formal solutions of the form:

$$
\hat{y}_{l}(z)=e^{q_{l}(z)} z^{\rho_{l}} \hat{h}_{l}(z)
$$

where $\rho_{l} \in \mathbf{C}, q_{l} \in z^{-1 / p} \mathbf{C}\left[z^{-1 / p}\right]$, and $\hat{h}_{l} \in \mathbf{C}\left[\left[z^{1 / p}\right]\right][\log z]$ for some $p \in \mathbf{N}$.

According to the main asymptotic existence theorem (cf. [26]) there exists $\epsilon>0$ such that for every interval $I \subset \mathbf{R}$ with length $|I|<\epsilon$ and every $l \in\{1, \ldots, n\}$, there exists a solution $y_{l, I}$ of $(D)$ with the property that $y_{l, I} \sim \hat{y}_{l}$ on $I$, i.e.

$$
e^{-q_{l}(z)} z^{-\rho_{l}} y_{l, I}(z) \sim \hat{h}_{l}(z) \text { as } z \rightarrow 0, \arg z \in I .
$$

In general, however, this solution is not unique. The object of exact asymptotics is to associate a unique "sum" with a given divergent power series. For the class of divergent power series arising in the theory of analytic differential equations this can be achieved by means of a process called 'multisummation'. There exist two, essentially equivalent, approaches to the theory of multi-summability: The analytic approach, which is based on the use of Borel and Laplace transformations, and the geometric approach. In the 
latter, an important part is played by so-called ( $k$-precise) quasi-functions: Analytic functions which are defined modulo functions with exponential decrease of some order $(k)$. The term $k$-precise quasi-function was introduced by J.-P. Ramis in [24]. In this paper, we explain and develop the second approach.

The paper is organized as follows. In $\S 1$ we give an elementary and constructive proof of the multi-summability of the formal series $\hat{h}_{l}$ in (1). This section serves as an introduction to and motivation of the geometric approach. In $\S 2$ we derive some basic properties of quasi-functions and multi-sums. $\S 3$ deals with Cauchy-Heine transforms in relation to multisummability and the Stokes phenomenon. Here, also the inverse problem is considered: To construct multi-sums with a prescribed Stokes phenomenon. In $\S 4$ we discuss the Stokes phenomenon in a fixed direction. In $\S 5$ we introduce Stokes automorphisms and give a definition of the alien derivations of J. Ecalle in terms of quasi-functions. The main ideas are illustrated in two very simple examples of linear differential equations.

\section{Introductory example.}

We begin by introducing some notation.

Definition 1.1. Let $I \subset \mathbf{R}$ be an open interval. $\mathcal{A}(I)$ is the set of all functions $f$ with the property that, for any closed interval $I^{\prime} \subset I$, there exists a positive number $r$ such that $f$ is holomorphic on the sector $S\left(I^{\prime}, r\right):=$ $\left\{z:|z|<r, \arg z \in I^{\prime}\right\}$ of the Riemann surface of $\log z$. With the natural restriction mapping, the sets $\mathcal{A}(I)$ form the sheaf $\mathcal{A}$ on $\mathbf{R}$ of holomorphic functions in a sector with vertex at $O$. For any $\theta \in I$, the germ at $\theta$ of $f \in \mathcal{A}(I)$ is the equivalence class of functions $f^{\prime} \in \mathcal{A}\left(I^{\prime}\right)$, where $I^{\prime} \ni \theta$, such that $\left.f^{\prime}\right|_{I^{\prime \prime}}=\left.f\right|_{I^{\prime \prime}}$ for some open interval $I^{\prime \prime} \subset I \cap I^{\prime}$ containing $\theta$. This germ is denoted by $f_{\theta}$. The stalk $\mathcal{A}_{\theta}$ of $\mathcal{A}$ is the set of all germs at $\theta$ of functions $f \in \mathcal{A}(I)$, where $I$ is any open interval containing $\theta$.

Let $k>0$. By $\mathcal{A}^{\leq-k}, \mathcal{A}^{<-k}, \mathcal{A}^{\leq k}$ and $\mathcal{A}^{<k}$ we denote the sheaves on $\mathbf{R}$ of holomorphic functions with at least exponential decrease of order $k$, with 'supra-exponential decrease of order $k$ ', with at most exponential growth of order $k$ and with 'subexponential growth of order $k$ ', respectively, in some sector with vertex at the origin. More precisely, if $I \subset \mathbf{R}$ is an open interval, $\mathcal{A}^{\leq-k}(I), \mathcal{A}^{<-k}(I), \mathcal{A}^{\leq k}(I)$ and $\mathcal{A}^{<k}(I)$ are the sets of all functions $f$ with the property that, for any closed interval $I^{\prime} \subset I$, there exists a positive number $r$ such that $f$ is holomorphic on the sector $S\left(I^{\prime}, r\right):=\{z:|z|<$ $\left.r, \arg z \in I^{\prime}\right\}$ of the Riemann surface of $\log z$, and

$$
\sup _{z \in S\left(I^{\prime}, r\right)}|f(z)| e^{c|z|^{-k}}<\infty
$$

for some $c>0$, for all $c>0$, for some $c<0$ and for all $c<0$, respectively. 
We consider the differential equation $(D)$ with formal solutions (1). For $l, m \in\{1, \ldots, n\}$, let $q_{m l}=q_{m}-q_{l}$. If $q_{l} \not \equiv q_{m}$, there exist $\kappa_{m l} \in \frac{1}{p} \mathbf{N}$ and $\omega_{m l} \in \mathbf{C}^{*}$ such that

$$
q_{m l}(z)=\omega_{m l} z^{-\kappa_{m l}}+o\left(z^{-\kappa_{m l}}\right) .
$$

Definition 1.2. The numbers $\kappa_{m l}$ are called the levels of the differential equation $(D)$. We say that $m \prec l$ on the interval $I$ if $e^{q_{m l}(z)}$ decreases exponentially of order $\kappa_{m l}$ as $z \rightarrow 0, \arg z \in I$ (so if $\cos \left(\arg \omega_{m l}-\kappa_{m l} \theta\right)<0$ if $\theta \in I) . \quad \theta$ is a Stokes direction of level $\kappa_{m l}$ for the pair $(m, l)$ if $\cos \left(\arg \omega_{m l}-\kappa_{m l} \theta\right)=0$. Let the increasing sequence of Stokes directions be denoted by $\left\{\theta_{h}\right\}_{h \in \mathbf{Z}}$. If $m \prec l$ on an interval $\left(\theta_{\sigma}, \theta_{\mu}\right)$ of length $\pi / \kappa_{m l}$ then $\left\{\theta_{\sigma}, \theta_{\mu}\right\}$ is called a $(m \prec l)$-Stokes pair or a Stokes pair of level $\kappa_{m l}$ and we denote $\theta_{\mu}$ by $\theta_{m l}$. If $I_{j}, j=1, \ldots, r$, are open intervals such that $I_{r} \subset I_{r-1} \subset \ldots \subset I_{1}$ then $\left(I_{1}, \ldots, I_{r}\right)$ will be called a nested multiinterval. If $I$ and $I^{(\nu)}, \nu=M, \ldots, N$, are open intervals where $M$ and $N$ are integers such that $M<N, \cup_{\nu=M}^{N} I^{(\nu)}=I$ and $I^{(\nu)} \cap I^{(\mu)} \neq \emptyset$ iff $|\nu-\mu| \leq 1$ then $\left\{I^{(\nu)}\right\}_{\nu=M}^{N}$ is called a finite covering of $I$.

Suppose that $(D)$ has $\mathrm{r}$ distinct levels: $k_{1}, \ldots, k_{r}$ in order of increasing magnitude. It is known that one can associate a unique sum to $\hat{y}_{l}$ and any nested multi-interval $\left(I_{1}, \ldots, I_{r}\right)$, if $\left|I_{j}\right|>\pi / k_{j}$ and $I_{j}$ does not contain Stokes pairs of level $k_{j}$ (cf. [22], [2], [5], [21]). Here we give another proof of this property based on the main asymptotic existence theorem mentioned above. Another proof based on this theorem has been given by M. van der Put (to appear). Similar ideas can be found in B. Malgrange [19]. From the main asymptotic existence theorem we first derive a fundamental system of normalized solutions, due in the generic case to G.D. Birkhoff $[\mathbf{3}]$ and in the general case to W. Balser, W.B. Jurkat and D.A. Lutz (cf. [13, p. 85]).

Lemma 1.3. Let $l \in\{1, \ldots, n\}, \nu_{0} \in \mathbf{Z}$. Then there exist solutions $\left\{y_{l}^{(\nu)}\right\}_{\nu \in \mathbf{Z}}$ of $(D)$ such that $y_{l}^{(\nu)} \sim \hat{y}_{l}$ on $\left(\theta_{\nu-1}, \theta_{\nu+1}\right)$ and

$$
\begin{aligned}
& y_{l}^{(\nu)}-y_{l}^{(\nu-1)}=\sum_{\theta_{h l}=\theta_{\nu}} \alpha_{h}(l, \nu) y_{h}^{(\nu-1)} \text { if } \nu>\nu_{0}, \\
& y_{l}^{(\nu)}-y_{l}^{(\nu-1)}=\sum_{\theta_{l h}=\theta_{\nu-1}} \alpha_{h}(l, \nu) y_{h}^{(\nu)} \text { if } \nu \leq \nu_{0} .
\end{aligned}
$$

Proof. Let $I:=(a, b)$ be an interval that contains a Stokes direction $\theta_{\nu}$ and with $|I|<\epsilon$ as in the Introduction. Then it is well known and easy to see that $y_{l, I} \sim \hat{y}_{l}$ on $\left(\theta_{\nu-1}, \theta_{\nu+1}\right)$. Denote $y_{l, I}$ by $\tilde{y}_{l}^{(\nu)}$.

Let $y_{l}^{\left(\nu_{0}\right)}:=\tilde{y}_{l}^{\left(\nu_{0}\right)}$ for $l=1, \ldots, n$. Let $\nu \geq \nu_{0}+1$ and suppose the construction of $y_{l}^{(\nu)}$ satisfying (2) has been performed up to $\nu-1$. Let 
$I=\left(\theta_{\nu-1}, \theta_{\nu+1}\right)$. Then there exist constants $b_{h}$ such that

$$
\tilde{y}_{l}^{(\nu)}-y_{l}^{(\nu-1)}=\sum_{\theta_{h l}=\theta_{\nu}} b_{h} y_{h}^{(\nu-1)}+\sum_{h \prec l \text { on } I} b_{h} \tilde{y}_{h}^{(\nu)},
$$

since the solutions $y_{h}^{(\nu-1)}$ with $\theta_{h l}=\theta_{\nu}$ and $\tilde{y}_{h}^{(\nu)}$ with $h \prec l$ on $I$ can be extended to a fundamental system by solutions $\tilde{y}_{h}^{(\nu)}$ which dominate the lefthand side on $\left(\theta_{\nu-1}, \theta_{\nu}\right)$. Therefore we choose

$$
y_{l}^{(\nu)}=\tilde{y}_{l}^{(\nu)}-\sum_{h \prec l \text { on } I} b_{h} \tilde{y}_{h}^{(\nu)}=y_{l}^{(\nu-1)}+\sum_{\theta_{h l}=\theta_{\nu}} b_{h} y_{h}^{(\nu-1)},
$$

so that $y_{l}^{(\nu)} \sim \hat{y}_{l}$ on $I$ and (2) holds up to $\nu$. Thus we recursively obtain all $y_{l}^{(\nu)}$ with $\nu \geq \nu_{0}$. Similarly the case with $\nu<\nu_{0}$ can be handled.

From this lemma we deduce:

Theorem 1.4. Let $l \in\{1, \ldots, n\}$ and $\left(I_{1}, \ldots, I_{r}\right)$ be a nested multi-interval such that $\left|I_{j}\right|>\pi / k_{j}$ and $I_{j}$ does not contain any $(h \prec l)$-Stokes pair of level $\kappa_{h l}=k_{j}$ for $j=1, \ldots, r$. Let $k_{r+1}:=\infty$. Then there exist a finite covering $\left\{I^{(\nu)}\right\}$ of $I_{1}$ and solutions $w_{l}^{(\nu)}$ of $(D)$ such that the endpoints of all $I_{j}$ belong to the set of endpoints of all $I^{(\nu)}$ and such that

$$
w_{l}^{(\nu)} \sim \hat{y}_{l} \text { on } I^{(\nu)},
$$

$$
e^{-q_{l}}\left(w_{l}^{(\nu)}-w_{l}^{(\nu-1)}\right) \in \mathcal{A}^{\leq-k_{j+1}}\left(I^{(\nu-1)} \cap I^{(\nu)}\right) \quad \text { if } I^{(\nu-1)} \cup I^{(\nu)} \subset I_{j} .
$$

Thus $e^{-q_{l}}\left\{w_{l}^{(\nu)}\right\}_{I^{(\nu)} \subset I_{j}}$ defines an element

(6) $e^{-q_{l}} w_{l j} \in \mathcal{A} / \mathcal{A}^{\leq-k_{j+1}}\left(I_{j}\right)$ with $e^{-q_{l}} w_{l j} \quad\left(\bmod \mathcal{A}^{\leq-k_{j}}\right)=\left.e^{-q_{l}} w_{l, j-1}\right|_{I_{j}}$.

Moreover, if $j \in\{1, \ldots, r\}$ then $w_{l j}$ is uniquely determined by $\hat{y}_{l}$ and the multi-interval $\left(I_{1}, \ldots, I_{j}\right)$. In particular, $w_{l r}$ is an ordinary function which is a uniquely determined solution of $(D)$ on $I_{r}$.

Proof. It is sufficient to give the proof for the case that the intervals $I_{j}$ have Stokes directions as endpoints since otherwise we can enlarge $I_{j}$ to such intervals with the other assumptions remaining satisfied. Let $I_{j}=$ $\left(a_{j}, b_{j}\right), j=1, \ldots, r$ and $I^{(\nu)}:=\left(\theta_{\nu-1}, \theta_{\nu+1}\right), \nu \in \mathbf{Z}$. We use Lemma 1.3 with $\nu>\nu_{0}$ where $\theta_{\nu_{0}}<a_{1}$. Let the linear operator $M_{j}^{(\nu)}$ on the solution space of $(D)$ be defined by

$$
M_{j}^{(\nu)} y_{m}^{(\nu)}=y_{m}^{(\nu-1)}+\sum_{\theta_{h m}=\theta_{\nu}, \kappa_{h m} \leq k_{j}} \alpha_{h}(m, \nu) y_{h}^{(\nu-1)}, m=1, \ldots, n,
$$


except if

$$
\theta_{\nu-1}=a_{p} \in I_{j}(\text { so } p>j) \text { and } \kappa_{m l}=k_{p}, m \prec l \text { on } I^{(\nu)}
$$

in which case we replace the righthand side of (7) by 0 . Then (2) implies that

(9)

$$
e^{-q_{l}}\left(1-M_{j}^{(\nu)}\right) y_{m}^{(\nu)} \in \mathcal{A}^{\leq-k_{j+1}}\left(I^{(\nu-1)} \cap I^{(\nu)}\right) \text { if } m=l \text { or } m \prec l \text { on } I^{(\nu)} .
$$

Define $w_{l}^{(\nu)}=y_{l}^{(\nu)}, \forall \nu \in \mathbf{Z}$ with $\theta_{\nu+1} \geq b_{1}$ and and from these by downward recursion in $\nu$ with $a_{1}<\theta_{\nu-1}<\theta_{\nu}<b_{1}$ :

$$
w_{l}^{(\nu-1)}=M_{j}^{(\nu)} w_{l}^{(\nu)} \text { if } j:=\max \left\{t:\left[\theta_{\nu-1}, \theta_{\nu}\right] \subset I_{t}\right\} .
$$

We will show that there exist constants $c_{m}(\nu)$ such that if $\theta_{\nu}>a_{1}$ :

$$
w_{l}^{(\nu)}-y_{l}^{(\nu)}=\sum_{m \in J(\nu)} c_{m}(\nu) y_{m}^{(\nu)}
$$

if

$$
J(\nu):=\left\{m \in\{1, \ldots, n\}: m \neq l, \kappa_{m l}=k_{p}, \theta_{\nu} \in I_{p}, m \prec l \text { on }\left(a_{p}, \theta_{\nu+1}\right)\right\} .
$$

If $m \in J(\nu)$ then $a_{p} \leq \theta_{\nu-1}$ and $m \prec l$ on $I^{(\nu)}$. Hence (4) follows from (11). Since $w_{l}^{(\nu)}-w_{l}^{(\nu-1)}=\left(1-M_{j}^{(\nu)}\right) w_{l}^{(\nu)}$ we obtain (5) from (11), (12) and (9).

Proof of (11). If $\theta_{\nu+1} \geq b_{1}$ then $w_{l}^{(\nu)}=y_{l}^{(\nu)}$ and (11) follows. Next suppose (11) holds for some $\nu$ with $\left[\theta_{\nu-1}, \theta_{\nu}\right] \subset I_{1}$. From (10) and (7) it follows that

$$
w_{l}^{(\nu-1)}-y_{l}^{(\nu-1)}=\sum_{\theta_{\lambda l}=\theta_{\nu}, \kappa_{\lambda l} \leq k_{j}} \alpha_{\lambda}(l, \nu) y_{\lambda}^{(\nu-1)}+\sum_{m \in J(\nu)} c_{m}(\nu) M_{j}^{(\nu)} y_{m}^{(\nu)},
$$

where

$$
\begin{gathered}
j:=\max \left\{t:\left[\theta_{\nu-1}, \theta_{\nu}\right] \subset I_{t}\right\}, \\
\kappa_{\lambda l}=k_{s}, s \leq j, \kappa_{m l}=k_{p}, \theta_{\nu} \in I_{p}, m \prec l \text { on }\left(a_{p}, \theta_{\nu+1}\right) .
\end{gathered}
$$

We only have to show that the righthand side of (13) is a linear combination of $y_{h}^{(\nu-1)}$ with $h \in J(\nu-1)$. As $\left[\theta_{\nu-1}, \theta_{\nu}\right] \subset I_{j} \subset I_{s}$ and $\left\{\theta_{\nu}-\pi / k_{s}, \theta_{\nu}\right\}$ is a $(\lambda \prec l)$-Stokes pair which by assumption is not included in $I_{s}$ we have $\theta_{\nu}-\pi / k_{s} \leq a_{s}$ and therefore $\lambda \prec l$ on $\left(a_{s}, \theta_{\nu}\right)$ and $\lambda \in J(\nu-1)$.

Remains to consider the terms $M_{j}^{(\nu)} y_{m}^{(\nu)}$ in (13), so with $m \in J(\nu)$. If $j<p$ then since $\theta_{\nu} \in I_{p}$, we have $\theta_{\nu-1} \notin I_{p}$ (cf. (14)). Therefore $\theta_{\nu-1}=a_{p}, m \prec l$ on $I^{(\nu)}$ (cf. (15)) and $M_{j}^{(\nu)} y_{m}^{(\nu)}=0$ (cf. (8)). Finally suppose $p \leq j$. Then 
$\theta_{\nu-1} \in I_{j} \subset I_{p}$ and $m \in J(\nu-1)$. We have to show that for $h$ in the sum in (7), so if $\theta_{h m}=\theta_{\nu}, \kappa_{h m}=k_{i}, i \leq j$, we have $h \in J(\nu-1)$. Now $h \prec m$ on $\left(\theta_{\nu}-\pi / k_{i}, \theta_{\nu}\right)$. From this and (15) we get $h \prec m \prec l$ on $\left(a, \theta_{\nu}\right)$, where $a:=\max \left\{\theta_{\nu}-\pi / k_{i}, a_{p}\right\}<\theta_{\nu}$. Since $q_{h l}=q_{h m}+q_{m l}$ we have $\kappa_{h l}=k_{\mu}$ where $\mu=\max \{i, p\}$. Then $\mu \leq j, a_{p} \leq a_{\mu} \leq a_{j}<\theta_{\nu}<b_{j} \leq b_{\mu}$. Hence the condition on Stokes pairs implies $a_{\mu} \geq \theta_{\nu}-\pi / k_{\mu} \geq \theta_{\nu}-\pi / k_{i}$ and so $a_{\mu} \geq a$. Therefore $h \prec l$ on $\left(a_{\mu}, \theta_{\nu}\right)$, i.e. $h \in J(\nu-1)$. Thus (11) holds in general.

The uniqueness statement follows from the next Proposition 1.5.

Proposition 1.5. Let $j \in\{1, \ldots, r\}, k:=k_{j}, m:=k_{j+1}$. Let $I$ be an open interval of length $>\frac{\pi}{k}$. Suppose there exist a finite covering $\left\{I^{(\nu)}\right\}_{\nu=1}^{N}$ of $I$ and solutions $u_{l}^{(\nu)}$ and $v_{l}^{(\nu)}$ of $(D), \nu=1, \ldots, N, l=1, \ldots, n$, satisfying the folowing conditions

1) $u_{l}^{(\nu)}(z) \sim \hat{y}_{l}(z)$ on $I^{(\nu)}$,

2) $e^{-q_{l}}\left(u_{l}^{(\nu)}-u_{l}^{(\nu-1)}\right) \in \mathcal{A}^{\leq-m}\left(I^{(\nu)} \cap I^{(\nu-1)}\right)$ if $\nu>1$,

3) $v_{l}^{(\nu)}$ satisfies the same conditions as $u_{l}^{(\nu)}$ and $e^{-q_{l}}\left(u_{l}^{(\nu)}-v_{l}^{(\nu)}\right) \in$ $\mathcal{A} \leq-k\left(I^{(\nu)}\right)$.

Then $e^{-q_{l}}\left(u_{l}^{(\nu)}-v_{l}^{(\nu)}\right) \in \mathcal{A}^{\leq-m}\left(I^{(\nu)}\right), \nu=1, \ldots, N$.

Proof. There exist constants $c_{h, \nu}$ such that

$$
v_{l}^{(\nu)}-u_{l}^{(\nu)}=\sum_{h \prec l \text { on } I^{(\nu)}, \kappa_{h l}=k} c_{h, \nu} u_{h}^{(\nu)} \quad\left(\bmod e^{q_{l}} \mathcal{A}^{\leq-m}\left(I^{(\nu)}\right)\right) .
$$

Hence if $\nu \geq 2$ then on $I^{(\nu)} \cap I^{(\nu-1)}$ we have

$$
\begin{aligned}
v_{l}^{(\nu-1)} & =v_{l}^{(\nu)} \quad\left(\bmod e^{q_{l}} \mathcal{A}^{\leq-m}\left(I^{(\nu)} \cap I^{(\nu-1)}\right)\right) \\
& =u_{l}^{(\nu)}+\sum_{h \prec l \text { on } I^{(\nu)}, \kappa_{h l}=k} c_{h, \nu} u_{h}^{(\nu)} \quad\left(\bmod e^{q_{l}} \mathcal{A}^{\leq-m}\left(I^{(\nu)} \cap I^{(\nu-1)}\right)\right) .
\end{aligned}
$$

In the last sum we have $u_{h}^{(\nu)}=u_{h}^{(\nu-1)}\left(\bmod e^{q_{h}} \mathcal{A} \leq-m\left(I^{(\nu)} \cap I^{(\nu-1)}\right)\right), h \prec l$ on $I^{(\nu)}$ and $\kappa_{h l}=k$. So using (16) with $\nu$ replaced by $\nu-1$ we see that $c_{h, \nu}=c_{h, \nu-1}$ and $c_{h, \nu} \neq 0$ only if $h \prec l$ on $I^{(\nu)} \cup I^{(\nu-1)}$. By varying $\nu$ we see that $c_{h, \nu} \neq 0$ only if $h \prec l$ on $I$ which is impossible since $\kappa_{h l}=k,|I|>\pi / k$. The assertions now follow from (16) with $c_{h, \nu}=0$.

Remark 1.6. $e^{-q_{l}} w_{l r}$ is called the multi-sum of $e^{-q_{l}} \hat{y}_{l}$ associated with the multi-interval $\left(I_{1}, \ldots, I_{r}\right)$. A generalization of Proposition 1.5 is Watson's lemma (cf. Proposition 2.3). Note that any interval $I_{j}$ in Theorem 1.4 contains a Stokes direction of level $k_{j}$. The construction in the proof of Theorem 1.4 only uses coverings by intervals $I^{(\nu)}=\left(\theta_{\nu-1}, \theta_{\nu+1}\right)$ and intervals $I_{j}$ bounded by Stokes directions. It could also be given with upward 
recursion by choosing $\theta_{\nu_{0}}>b_{1}$ in Lemma 1.3. If $y_{l}^{(\nu)}$ is a normalized solution in the sense of Jurkat (cf. Lemma 1.3) with $\theta_{\nu+1}-\theta_{\nu-1}>\pi / k_{r}$ then the proof of Theorem 1.4 implies that $e^{-q_{l}} y_{l}^{(\nu)}$ is the multisum of $e^{-q_{l}} \hat{y}_{l}$ on nested multi-intervals $\left(I_{1}, \ldots, I_{r}\right)$ where $\left|I_{j}\right|>\pi / k_{j}, I_{j}$ does not contain any $(h \prec l)$-Stokes pair of level $\kappa_{h l}=k_{j}$ and either $I_{j}=\left(a_{j}, \theta_{\nu+1}\right), j=$ $1, \ldots, r, a_{r}=\theta_{\nu-1}$ or $I_{j}=\left(\theta_{\nu-1}, b_{j}\right), j=1, \ldots, r, b_{r}=\theta_{\nu+1}$.

Next we consider the Stokes phenomenon for multi-sums of formal solutions of $(D)$. As we have seen above it is sufficient to consider multisums on intervals bounded by Stokes directions.

Proposition 1.7. Let $l \in\{1, \ldots, r\}$ and let $\left(I_{1}, \ldots, I_{r}\right)$ and $\left(\tilde{I}_{1}, \ldots, \tilde{I}_{r}\right)$ be nested multi-intervals such that if $j \in\{1, \ldots, r\}$ then $I_{j}$ and $\tilde{I}_{j}$ have length $>\pi / k_{j}$ and they do not contain any Stokes pair of level $k_{j}$. Moreover, assume that there exist $i \in\{1, \ldots, r\}$ with the property $I_{j}=\tilde{I}_{j}$ if $j \neq i$, and $I_{i} \cap \tilde{I}_{i}=\left(\theta_{\sigma}, \theta_{\mu}\right)$ such that $\theta_{\sigma} \in I_{i}$ and $\left\{\theta_{\sigma}, \theta_{\mu}\right\}$ is a $(s \prec l)$-Stokes pair of level $\kappa_{s l}=k_{i}$ for some $s \in\{1, \ldots, r\}$. Let $\Lambda:=\left\{h: \theta_{h l}=\theta_{\mu}, \kappa_{h l}=k_{i}\right\}$ and let $u_{l j}$ and $v_{l j}, j=1, \ldots, r$ satisfy the properties of Theorem 1.4 with respect to $\left(I_{1}, \ldots, I_{r}\right)$ and $\left(\tilde{I}_{1}, \ldots, \tilde{I}_{r}\right)$ respectively.

Then there exist constants $\gamma_{h}, h \in \Lambda$ such that

$$
v_{l j}-u_{l j}=\sum_{h \in \Lambda} \gamma_{h} u_{h j} \text { if } j \geq i \text {. }
$$

Proof. We may assume that $u_{l}^{(\nu)}$ and $v_{l}^{(\nu)}$ are constructed as in the proof of Theorem 1.4. Let $I_{i}=\left(\theta_{\alpha}, \theta_{\mu}\right)$ and $\tilde{I}_{i}=\left(\theta_{\sigma}, \theta_{\beta}\right)$. Then $\alpha<\sigma<$ $\mu<\beta,\left(\theta_{\alpha}, \theta_{\beta}\right) \subset I_{i-1}$ and if $i<r$ also $I_{i+1} \subset\left(\theta_{\sigma}, \theta_{\mu}\right)$ and $\sigma<\mu-1$. Let $j:=\max \left\{s: \theta_{\nu-1}, \theta_{\nu} \in I_{s}\right\}, \tilde{j}:=\max \left\{s: \theta_{\nu-1}, \theta_{\nu} \in \tilde{I}_{s}\right\}$. If $\nu \geq \beta$ then $j<i, \tilde{j}<i$ and therefore $j=\tilde{j}$. From (10) it now follows that $v_{l}^{(\nu)}=u_{l}^{(\nu)}$ if $\nu \geq \beta-1$.

Let $\nu:=\min \left\{\lambda \geq \mu: v_{l}^{(\lambda)}=u_{l}^{(\lambda)}\right\}$. So $\mu \leq \nu \leq \beta-1, \theta_{\nu} \in \tilde{I}_{i} \backslash I_{i}$. Then $j=i-1$, and except in case $\nu=\mu=\sigma+1$ moreover $\tilde{j}=i$. From (10) and (11) it follows that except in case $\nu=\mu=\sigma+1$ :

$$
\begin{aligned}
v_{l}^{(\nu-1)}-u_{l}^{(\nu-1)} & =\left(M_{i}^{(\nu)}-M_{i-1}^{(\nu)}\right) u_{l}^{(\nu)} \\
& =\left(M_{i}^{(\nu)}-M_{i-1}^{(\nu)}\right)\left(y_{l}^{(\nu)}+\sum_{m \in J_{g}(\nu)} c_{m}(\nu) y_{m}^{(\nu)}\right)
\end{aligned}
$$

with $g=\max \left\{t: \theta_{\nu}, \theta_{\nu+1} \in I_{t}\right\}$. Then $g<i$ as $\theta_{\nu} \notin I_{i}$. From (7) we get

$$
\left(M_{i}^{(\nu)}-M_{i-1}^{(\nu)}\right) y_{m}^{(\nu)}=\sum_{\theta_{h m}=\theta_{\nu}, \kappa_{h m}=k_{i}} \alpha_{h}(m, \nu) y_{h}^{(\nu-1)} .
$$


If $m \in J_{g}(\nu), \kappa_{m l}=k_{p}, \kappa_{h m}=k_{i}, \theta_{h m}=\theta_{\nu}$ then $p \leq g<i$ and $m \prec l$ on $\left(a_{p}, \theta_{\nu+1}\right)$. As $q_{h l}=q_{h m}+q_{m l}$ we have $\kappa_{h l}=k_{i}, \theta_{h l}=\theta_{\nu}$. Since $\theta_{\nu} \in \tilde{I}_{i}$ we have $\theta_{\nu}-\pi / k_{i} \notin \tilde{I}_{i}=\left(\theta_{\mu}-\pi / k_{i}, \theta_{\beta}\right)$ and as $\nu \geq \mu$ we get $\nu=\mu$. Hence $v_{l}^{(\nu)}=u_{l}^{(\nu)}$ if $\nu \geq \mu$. So if $\mu>\sigma+1$ we get:

$$
v_{l}^{(\mu-1)}-u_{l}^{(\mu-1)}=\sum_{h \in \Lambda} \delta_{h} y_{h}^{(\mu-1)} .
$$

Next we consider (11) for $u_{h}^{(\mu-1)}$ :

$$
u_{h}^{(\mu-1)}=y_{h}^{(\mu-1)}+\sum_{m \in J_{h i}(\mu-1)} c_{m h}(\mu-1) y_{m}^{(\mu-1)}
$$

where $J_{h i}(\mu-1):=\left\{m: m \prec h\right.$ on $\left.\left(a_{s}, \theta_{\mu}\right), \kappa_{m h}=k_{s}, s \leq i\right\}$. If $h \in \Lambda, m \in$ $J_{h i}(\mu-1)$ then $m \in \Lambda$ by the same argument as above. Since the solutions $u_{h}^{(\mu-1)}, h \in \Lambda$, are linearly independent and the same holds for $y_{h}^{(\mu-1)}$, we obtain

$$
v_{l}^{(\nu)}-u_{l}^{(\nu)}=\sum_{h \in \Lambda} \gamma_{h} u_{h}^{(\nu)} \quad\left(\bmod e^{q_{l}} \mathcal{A}^{\leq-k_{j+1}}\left(I^{(\nu)}\right)\right)
$$

for $\nu=\mu-1, j=i$. From the construction of $u_{l}^{(\nu)}$ and $v_{l}^{(\nu)}$ for $\nu<\mu-1$ by means of (10) we now deduce (19) for $I^{(\nu)} \subset I_{j} \cap \tilde{I}_{j}, j \geq i$ if $\mu>\sigma+1$ and (17) follows.

In case $\nu=\mu=\sigma+1$ we have $i=r, j=r-1$ and thus $v_{l}^{(\mu)}-u_{l}^{(\mu-1)}=$ $\left(1-M_{r-1}^{(\mu)}\right) u_{l}^{(\mu)}=\left(1-M_{r-1}^{(\mu)}\right)\left(y_{l}^{(\mu)}+\sum_{m \in J_{r-1}(\mu)} c_{m}(\mu) y_{m}^{(\mu)}\right)$. From this and (7) we deduce in the same way as above $v_{l}^{(\mu)}-u_{l}^{(\mu-1)}=\sum_{h \in \Lambda} \gamma_{h} u_{h}^{(\mu-1)}$ which implies (17) since $v_{l r}=v_{l}^{(\mu)}$ and $u_{l r}=u_{l}^{(\mu-1)}$.

Remark 1.8. Proposition 1.7 shows that the only contributions to the Stokes phenomenon come from solutions which have as exponential factor $\exp q_{h}$ such that $\left\{\theta_{\sigma}, \theta_{\mu}\right\}$ is a $(h \prec l)$-Stokes pair of level $\kappa_{h l}=k_{i}$. So $\exp q_{h l}$ has maximal descent near 0 of order $k_{i}$ in the direction $\theta_{\mu}-\pi /\left(2 k_{i}\right)$, a so-called singular or anti-Stokes direction of level $k_{i}$ (cf. [5], [17]). For calculations of the Stokes multipliers see [5], [6] and Example 5.10.

The Stokes phenomenon of level $k_{i}$ occurs in general if in Proposition 1.7 the condition on $I_{i} \cap \tilde{I}_{i}$ is replaced by: $I_{i} \cup \tilde{I}_{i}$ contains exactly one Stokes pair $\left\{\theta_{\sigma}, \theta_{\mu}\right\}$ of level $k_{i}$ and this is a $(s \prec l)$-Stokes pair for some $s, \theta_{\sigma} \in I_{i}$. Without affecting the multisums we may replace $I_{i}$ by $\left(\theta_{\alpha}, \theta_{\mu}\right)$ and $\tilde{I}_{i}$ by $\left(\theta_{\sigma}, \theta_{\beta}\right)$ with suitable $\alpha, \beta$ such that $\alpha<\sigma, \mu<\beta$, in view of Proposition 1.5. Now again Proposition 1.7 is applicable. The case where there are more than one $i$ with $I_{i} \neq \tilde{I}_{i}$ can be reduced to the case with a single such $i$. 


\section{Some basic properties of quasi-functions and multisums.}

In this section we introduce some notations that will be used throughout the paper. Unfortunately, there is no uniformity of notation in the existing literature on Gevrey series and functions and the like. Furthermore, we recall a number of known properties of quasi-functions and multi-sums and prove some new results of a rather technical nature (cf. [1], [21], [20], [24]).

Definition 2.1. By $\mathcal{A}^{(0)}$ we denote the sheaf on $\mathbf{R}$ of holomorphic functions admitting an asymptotic expansion $\in \mathbf{C}[[z]]$ as $z \rightarrow 0$ in some sector with vertex at $O$ and by $\mathcal{A}^{(k)}$ the subsheaf of functions that are Gevrey of order $\frac{1}{k}$. If $f \in \mathcal{A}^{(0)}$ its asymptotic expansion will be denoted by $\hat{f}$ or $J(f)$. By $\mathcal{A}^{<0}$ we denote the kernel of the map $J: \mathcal{A}^{(0)} \rightarrow \mathbf{C}[[z]]$.

By $\mathbf{C}[[z]]_{k}$ we denote the set of formal power series that are Gevrey of order $k^{-1}$.

Let $k>0$ and let $I$ be an interval of $\mathbf{R}$, open, half-open or closed. A $k$-precise quasi-function $f$ on $I$ is a section on $I$ of the quotient-sheaf $\mathcal{Q}_{k}:=$ $\mathcal{A} / \mathcal{A}^{\leq-k}$, i.e. $f \in \mathcal{Q}_{k}(I)=\mathcal{A} / \mathcal{A}^{\leq-k}(I)$. A $k$-precise quasi-function $f$ can be represented by a collection of functions $\left\{f_{j}\right\}_{j \in \mathcal{J}}$ defined and analytic on an open covering $\left\{I_{j}\right\}_{j \in \mathcal{J}}$ of $I$. Two such collections $\left\{f_{j}\right\}_{j \in \mathcal{J}}$ defined on a covering $\left\{I_{j}\right\}_{j \in \mathcal{J}}$ and $\left\{f_{j}^{\prime}\right\}_{j \in \mathcal{J}^{\prime}}$ defined on a covering $\left\{I_{j}^{\prime}\right\}_{j \in \mathcal{J}^{\prime}}$ are considered equivalent if $\left.f_{j}\right|_{I_{j} \cap I_{h}^{\prime}}-\left.f_{h}^{\prime}\right|_{I_{j} \cap I_{h}^{\prime}} \in \mathcal{A}^{\leq-k}\left(I_{j} \cap I_{h}^{\prime}\right)$ for all $j \in \mathcal{J}$ and $h \in \mathcal{J}^{\prime}$ such that $I_{j} \cap I_{h}^{\prime} \neq \emptyset$. We say that $f$ is bounded, has exponential growth of order $l>0$, etc. if the functions $f_{j}, j \in \mathcal{J}$ have this property. If, for all $j \in \mathcal{J}, f_{j} \in \mathcal{A}^{(k)}\left(I_{j}\right)$ then the asymptotic expansion of $f_{j}$ is independent of $j$. This asymptotic expansion will again be denoted by $\hat{f}$ or $J(f)$.

$k$-precise quasi-functions with at most subexponential growth of order $k$, i.e. sections of the sheaf $\mathcal{Q}_{k}^{<k}:=\mathcal{A}^{<k} / \mathcal{A}^{\leq-k}$, have a property of analytic continuation. The following proposition is due to Malgrange [19, "quasianalyticité relative"]. It can be derived from Proposition 2.4 below.

Proposition 2.2. Let $k>0, I$ an interval of $\mathbf{R}$ and $f \in \mathcal{Q}_{k}^{<k}(I)$. If the germ of $f$ vanishes in a point $\theta$ of $I$ then $f=0$. More generally, the result also holds if we replace the condition that $f$ vanishes in $\theta$ by: On a neighborhood $I_{0}$ of $\theta f$ is represented by a function which has exponential decrease of order $k$ in the direction $\theta$.

Proposition 2.3. Let $f \in \mathcal{A}^{\leq-k} / \mathcal{A}^{<-k}(I)$ where $k>0$ and $I$ is an interval with length $>\pi / k$. Then $f=0$.

This proposition extends a result for ordinary functions (i.e. $f \in$ $\mathcal{A}^{\leq-k}(I)$ ), due to Watson. In the present form it can be found in MalgrangeRamis [21] (lemme de Watson relatif) and Kostov [15] (cf. also Il'yashenko [11]). We give another proof using the following proposition: 
Proposition 2.4. Let $f$ be holomorphic and of exponential type in the neighborhood of $\infty$ in a sector $S$ with vertex 0 . Let the extended indicator function $H_{f}: S \rightarrow[-\infty, \infty)$ be defined by $H_{f}(z):=\limsup _{r \rightarrow \infty} r^{-1} \log |f(r z)|$ if $z \in S$. Then $H_{f}$ is homogeneous of order 1 and convex on $S$. In particular: If the segment $\left[z_{1}, z_{2}\right] \subset S$ and $H_{f}\left(z_{j}\right)<A_{j}$ where $A_{j} \in \mathbf{R}, j=1,2$, then

$$
f\left(r\left(\lambda z_{1}+(1-\lambda) z_{2}\right)\right)=O(1) \exp \left\{r\left(\lambda A_{1}+(1-\lambda) A_{2}\right)\right\}
$$

as $r \rightarrow \infty$, uniformly in $\lambda \in[0,1]$.

This result can be deduced from the Phragmén-Lindelöf theorem as has been shown by E.G.F. Thomas (private communication). It also follows from the subharmonicity and homogeneity of $H_{f}$ (cf. [10, p. 313]). The restriction of $H_{f}$ to the unit circle is the usual indicator function.

Next consider the case that $f \in \mathcal{A}^{\leq 1} / \mathcal{A}^{<-1}(I)$ where $I$ is an interval $(a, b)$. Then we may define the extended indicator function for $f$ as follows. Let $I_{j}=\left(a_{j}, b_{j}\right), j=1,2$ be intervals with nonempty intersection and such that $f$ may be represented by functions $f_{j} \in \mathcal{A}^{\leq 1}\left(I_{j}\right)$ on $I_{j}$. Let $g_{j}(z)=f_{j}\left(z^{-1}\right)$ on a neighborhood of $\infty$ in the sector $S_{j}:-b_{j}<\arg z<-a_{j}$. Then the extended indicator function $H_{g_{j}}$ is convex on $S_{j}$ and moreover $H_{g_{1}}=H_{g_{2}}$ on $S_{1} \cap S_{2}$ since $g_{1}-g_{2} \in \mathcal{A}^{<-1}\left(S_{1} \cap S_{2}\right)$ (cf. [15]). Thus we obtain a uniquely defined function on the sector $S:-b<\arg z<-a$ which is convex on overlapping subsectors of $S$ and hence is convex on $S$. Moreover it is homogeneous of order 1 . We denote this function by $\tilde{H}_{f}$.

Proof of Proposition 2.3. We may assume $k=1$ without loss of generality. Then $\tilde{H}_{f}$ is defined on a sector $S$ of opening $>\pi$ and $\tilde{H}_{f}$ is negative and convex on $S$. From this it follows that $\tilde{H}_{f}$ has to be a negative constant on lines which belong entirely to $S$. Since any pair of points in $S$ can be connected by segments on such lines it follows that $\tilde{H}_{f}$ is a negative constant in $S$. Because $\tilde{H}_{f}$ is homogeneous of degree 1 this has to be $-\infty$. So by proposition 2.4 each of the representatives of $f$ has supra-exponential decrease of order 1 and therefore $f=0$.

Proof of Proposition 2.2. We may assume that $k=1$. Suppose that $f$ is represented by a function $f_{0}$ on $I_{0}$. Let $S_{0}$ be the sector with $z \in S_{0}$ iff $\arg z \in I_{0}$. Then $\tilde{H}_{f_{0}}$ is negative in the direction $-\theta$ and since it is nonpositive on $S_{0}$ (by the assumption) and convex it has to be negative on $S_{0}$. Hence $\left.f\right|_{I_{0}} \in \mathcal{A}^{\leq-1}\left(I_{0}\right)$. Repeating this reasoning we obtain a chain of subintervals of $I$ on which the representatives of $f$ are sections of $\mathcal{A}^{\leq-1}$. So $f=0$.

Lemma 2.5 ("Borel-Ritt", cf. [21]). The mapping $J: \mathcal{A}^{(0)} / \mathcal{A}^{<0} \rightarrow \mathbf{C}[[z]]$ is an isomorphism of sheaves of differential algebras and so is its "restriction" $J: \mathcal{A}^{(k)} / \mathcal{A}^{\leq-k} \rightarrow \mathbf{C}[[z]]_{k}$. 
Definition 2.6. Let $r \in \mathbf{N}, r>1$, let $0<k_{1}<\cdots<k_{r}$ and $\mathbf{k}=$ $\left(k_{1}, \ldots, k_{r}\right)$. For $j=1, \ldots, r$, let $I_{j}$ be an open, half-open or closed interval of $\mathbf{R}$. If $I_{r} \subset \cdots \subset I_{1}$ we call $\mathbf{I}:=\left(I_{1}, \ldots, I_{r}\right)$ a nested multi-interval. Let $f_{j}$ be a $k_{j}$-precise quasifunction on $I_{j}: f_{j} \in \mathcal{Q}_{k_{j}}\left(I_{j}\right), j \in\{1, \ldots, r\}$. If, for all $j \in\{2, \ldots, r\}$,

$$
f_{j} \quad\left(\bmod \mathcal{A}^{\leq-k_{j-1}}\right)=\left.f_{j-1}\right|_{I_{j}}
$$

we call $\mathbf{f}:=\left(f_{1}, \ldots, f_{r}\right)$ a $\mathbf{k}$-precise (or $\left(k_{1}, \ldots, k_{r}\right)$-precise) quasifunction on the nested multi-interval $\mathbf{I}$. The set of all $\mathbf{k}$-precise quasifunctions on I will be denoted by $\mathcal{Q}_{\left(k_{1}, \ldots, k_{r}\right)}\left(I_{1}, \ldots, I_{r}\right)$ or $\mathcal{Q}_{\mathbf{k}}(\mathbf{I})$. By $\mathcal{Q}_{\mathbf{k}}^{<l}(\mathbf{I}), \mathcal{Q}_{\mathbf{k}}^{\leq-l}(\mathbf{I})$, $\mathcal{Q}_{\mathbf{k}}^{(l)}(\mathbf{I})$, etc. we denote the sets of $\mathbf{k}$-precise quasifunctions on $\mathbf{I}$ that have subexponential growth of order $l$, exponential decrease of order $l$, that are Gevrey of order $1 / l$, etc.

Definition 2.7. Let $r \in \mathbf{N}$, let $0<k_{1}<\cdots<k_{r+1}=\infty$. Let $\hat{f} \in \mathbf{C}[[z]]_{k_{1}}$ and $f_{0}=J^{-1}(\hat{f})$. For $j=1, \ldots, r$ let $I_{j}$ be an open interval of $\mathbf{R}$ of length $>\frac{\pi}{k_{j}}$, or a half-open or closed interval of length $\geq \frac{\pi}{k_{j}}$, such that $I_{r} \subset \ldots \subset I_{1}$. If there exists $\mathbf{f}=\left(f_{1}, \ldots, f_{r}\right) \in \mathcal{Q}_{\left(k_{2}, \ldots, k_{r+1}\right)}^{\left(k_{1}\right)}\left(I_{1}, \ldots, I_{r}\right)$ such that $f_{1}\left(\bmod \mathcal{A}^{\leq-k_{1}}\right)=\left.f_{0}\right|_{I_{1}}$, the formal power series $\hat{f}$ is $\left(k_{1}, \ldots, k_{r}\right)$ summable on $\left(I_{1}, \ldots, I_{r}\right)$ with $\left(k_{1}, \ldots, k_{r}\right)$-sum (or multi-sum) $\left(f_{1}, \ldots, f_{r}\right)$. The set of all $\left(k_{1}, \ldots, k_{r}\right)$-sums on $\left(I_{1}, \ldots, I_{r}\right)$ will be denoted by $\Sigma_{\mathbf{k}}(\mathbf{I})$, where $\mathbf{k}=\left(k_{1}, \ldots, k_{r}\right)$ and $\mathbf{I}=\left(I_{1}, \ldots, I_{r}\right)$.

A formal series $\hat{f}$ of the form

$$
\hat{f}(z)=\sum_{\alpha \in \mathcal{J}} \sum_{l=0}^{p} \hat{f}^{\alpha l}(z) z^{\alpha}(\log z)^{l}
$$

where $p \in \mathbf{N}, \mathcal{J}$ is a finite subset of $\mathbf{C}$ and $\hat{f}^{\alpha l} \in \mathbf{C}[[z]]$ for all $l$ and $\alpha$, will be called $\mathbf{k}$-summable on the multi-interval $\mathbf{I}$, with $\mathbf{k}$-sum $\left(f_{1}, \ldots, f_{r}\right)$, if $\hat{f}^{\alpha l}$ is $\mathbf{k}$-summable on the multi-interval $\mathbf{I}$ for each $\alpha \in \mathcal{J}$ and each $l \in\{1, \ldots, p\}$, with k-sum $\left(f_{1}^{\alpha l}, \ldots, f_{r}^{\alpha l}\right)$, such that

$$
f_{j}(z)=\sum_{\alpha \in \mathcal{J}} \sum_{l=0}^{p} f_{j}^{\alpha l}(z) z^{\alpha}(\log z)^{l}, \quad j=1, \ldots, r .
$$

Let $d_{j} \in \mathbf{R}, j=1, \ldots, r . \mathbf{d}:=\left(d_{1}, \ldots, d_{r}\right)$ is called an admissible multidirection with respect to $\mathbf{k}$ if, for $j=2, \ldots, r$,

$$
\left|d_{j}-d_{j-1}\right| \leq \frac{\pi}{2 k_{j-1}}-\frac{\pi}{2 k_{j}}
$$

$\hat{f}$ is $\mathbf{k}$-summable in the multi-direction $\mathbf{d}$ if $\hat{f}$ is $\mathbf{k}$-summable on the multiinterval $\left(I_{1}, \ldots, I_{r}\right)$, where $I_{j}=\left[d_{j}-\frac{\pi}{2 k_{j}}, d_{j}+\frac{\pi}{2 k_{j}}\right]$. By $\mathbf{C}\{z\}_{\mathbf{k}, \mathbf{d}}$ we denote 
the set of all formal power series that are $\mathbf{k}$-summable in the multi-direction d.

An admissible multi-direction $\mathbf{d} \in \mathbf{R}^{r}$ is called a singular direction of $\hat{f}$ if $\hat{f} \notin \mathbf{C}\{z\}_{\mathbf{k}, \mathbf{d}}$.

Let $\hat{f} \in \mathbf{C}[[z]]_{k_{1}}$ and $f_{0}=J^{-1}(\hat{f})$ and suppose there exist $s \in \mathbf{N}$ and, if $s>1$, positive numbers $k_{1}<\cdots<k_{s}$, an admissible multi-direction $\left(d_{1}, \ldots, d_{s}\right)$ with respect to $\left(k_{1}, \ldots, k_{s}\right)$ and $\left(f_{1}, \ldots, f_{s-1}\right) \in \mathcal{Q}_{\left(k_{2}, \ldots, k_{s}\right)}\left(I_{1}, \ldots, I_{s-1}\right)$, where $I_{j}=\left[d_{j}-\frac{\pi}{2 k_{j}}, d_{j}+\frac{\pi}{2 k_{j}}\right], j=1, \ldots, s-1$, such that $f_{1}\left(\bmod \mathcal{A}^{\leq-k_{1}}\right)=$ $\left.f_{0}\right|_{I_{1}}$. $d_{s}$ is called a singular direction of level $k_{s}$ of $\hat{f}$ if $\hat{f} \notin \mathbf{C}\{z\}_{\mathbf{k}^{\prime}, \mathbf{d}^{\prime}}$ for any $\mathbf{k}^{\prime}=\left(k_{1}^{\prime}, \ldots, k_{r}^{\prime}\right)$ with $r \geq s$ and any admissible multi-direction $\mathbf{d}^{\prime}=\left(d_{1}^{\prime}, \ldots, d_{r}^{\prime}\right)$ such that $k_{j}^{\prime}=k_{j}$ and $d_{j}^{\prime}=d_{j}$ for $j=1, \ldots, s$.

Let $\hat{f} \in \mathbf{C}[[z]], k>0$ and $\theta \in \mathbf{R}$. The pair $\left\{\theta, \theta+\frac{\pi}{k}\right\}$ will be called a Stokes pair of level $k$ for $\hat{f}$ if the number $\theta+\frac{\pi}{2 k}$ is a singular direction of level $k$ for $\hat{f}$. By $\mathbf{C}\{z\}_{\mathbf{k}}$ we denote the set of all $\hat{f} \in \mathbf{C}[[z]]$ with the property that $\hat{f} \in \mathbf{C}\{z\}_{\mathbf{k}, \mathbf{d}}$ for every admissible multi-direction $\mathbf{d}=\left(d_{1}, \ldots, d_{r}\right) \in \mathbf{R}^{r}$, except for a finite number of values of each single direction $d_{1}, \ldots, d_{r}$.

If $\hat{f}$ is $\left(k_{1}, \ldots, k_{r}\right)$-summable with $\left(k_{1}, \ldots, k_{r}\right)$-sum $\mathbf{f}=\left(f_{1}, \ldots, f_{r}\right)$, we say that $\hat{f}(\bmod \mathbf{C}\{z\})$ is $\left(k_{1}, \ldots, k_{r}\right)$-summable with $\left(k_{1}, \ldots, k_{r}\right)$-sum $\mathbf{f}$ $(\bmod \mathbf{C}\{z\})$.

The above definition of $\mathbf{k}$-summability is easily seen to be equivalent to the following.

Definition 2.8. Let $\mathbf{k}:=\left(k_{1}, \ldots, k_{r}\right)$ with $0<k_{1}<\ldots<k_{r}$ and $\mathbf{I}:=$ $\left(I_{1}, \ldots, I_{r}\right)$ a nested multi-interval and let $I_{0}=\mathbf{R}$. The formal power series $\hat{f} \in \mathbf{C}[[z]]_{k_{1}}$ is $\mathbf{k}$-summable on $\mathbf{I}$ if $f_{0}=J^{-1}(\hat{f})$ has a representative $\left\{f_{0, \nu}\right\}$ defined on a covering $\left\{I_{0, \nu}\right\}$ of $\mathbf{R}$, with the property that $f_{0, \nu}-f_{0, \nu+1} \in$ $\mathcal{A}^{\leq-k_{j+1}}\left(I_{0, \nu} \cap I_{0, \nu+1}\right)$ for all $\nu$ such that $I_{0, \nu} \cup I_{0, \nu+1} \subset I_{j}$.

Remark 2.9. Obviously, if $\left(f_{1}, \ldots, f_{r}\right)$ is a $\left(k_{1}, \ldots, k_{r}\right)$-sum on $\left(I_{1}, \ldots, I_{r}\right)$, then $f_{j} \in \mathcal{Q}_{k_{j+1}}^{\left(k_{1}\right)}\left(I_{j}\right)$ for all $j \in\{1, \ldots, r\}$. Suppose that $\left(f_{1}^{\prime}, \ldots, f_{r-1}^{\prime}, f_{r}\right)$ is another $\left(k_{1}, \ldots, k_{r}\right)$-sum on $\left(I_{1}, \ldots, I_{r}\right)$. Then we have, for all $j \in$ $\{0, \ldots, r\}$,

$$
\left.f_{j}\right|_{I_{r}}=\left.f_{j}^{\prime}\right|_{I_{r}}=f_{r} \quad\left(\bmod \mathcal{A}^{\leq-k_{j+1}}\right) .
$$

By Proposition 2.2, this implies that $f_{j}=f_{j}^{\prime}$ for all $j \in\{0, \ldots, r\}$. Thus the quasi-functions $f_{0}, \ldots, f_{r-1}$ are uniquely determined by $f_{r}$. Therefore, we will sometimes simply denote the multi-sum $\left(f_{1}, \ldots, f_{r}\right)$ by $f_{r}$. More generally, suppose that $\mathbf{I}=\left(I_{1}, \ldots, I_{r}\right)$ is a nested multi-interval, $\mathbf{k}=\left(k_{1}, \ldots, k_{r}\right)$ with $0<k_{1}<\cdots<k_{r}$ and $\left(f_{1}, \ldots, f_{r}\right) \in \mathcal{Q}_{\mathbf{k}}^{<k_{1}}(\mathbf{I})$. Then the quasifunctions $f_{1}, \ldots, f_{r}$ are uniquely determined by $f_{r}$.

Let $0<k_{1}<\cdots<k_{r}$. Suppose we are given a formal power series $\hat{f}$ and, for every $j \in\{1, \ldots, r\}$, an interval $\left(\alpha_{j}, \beta_{j}\right) \subset \mathbf{R}$. The following statements 
are equivalent: (i) $\left(\alpha_{j}, \beta_{j}\right)$ contains no singular direction of level $k_{j}$ for $\hat{f}$, and (ii) $\left(\alpha_{j}-\frac{\pi}{2 k_{j}}, \beta_{j}+\frac{\pi}{2 k_{j}}\right)$ contains no Stokes pair of level $k_{j}$ for $\hat{f}$. This can be deduced from Lemma 2.11 below. The next lemma is an immediate consequence of this fact.

Lemma 2.10. Let $r \in \mathbf{N}, 0<k_{1}<\cdots<k_{r+1}=\infty$ and let $\left(I_{1}, \ldots, I_{r}\right)$ be a nested multi-interval with the property that $\left|I_{j}\right|>\frac{\pi}{k_{j}}$ for $j=1, \ldots, r$. A formal power series $\hat{f}$ is $\left(k_{1}, \ldots, k_{r}\right)$-summable on $\left(I_{1}, \ldots, I_{r}\right)$ if and only if, for $j=1, \ldots, r, I_{j}$ does not contain any Stokes pair of level $k_{j}$ for $\hat{f}$.

Lemma 2.11. Let $r \in \mathbf{N}$, let $0<k_{1}<\cdots<k_{r+1}=\infty$. Let $I_{j}, j=$ $0, \ldots, r$, be open intervals of $\mathbf{R}$ with the property that $I_{r} \subset I_{r-1} \subset \ldots \subset I_{0}$. For $j=0, \ldots, r$, let $\left\{I_{j}^{i}\right\}_{i \in \mathcal{J}}$ be an open covering of $I_{j}$ such that $I_{r}^{i} \subset$ $I_{r-1}^{i} \subset \ldots \subset I_{0}^{i}$ for all $i \in \mathcal{J}$, where $\mathcal{J}$ is an index set. Moreover, assume that $I_{1}^{i_{1}} \cap I_{1}^{i_{2}} \neq \emptyset$ for some $i_{1}, i_{2} \in \mathcal{J}$ implies $I_{r}^{i_{1}} \cap I_{r}^{i_{2}} \neq \emptyset$. Suppose there exist $f^{i}=\left(f_{0}^{i}, \ldots, f_{r}^{i}\right) \in \mathcal{Q}_{\left(k_{1}, \ldots, k_{r+1}\right)}^{<k_{1}}\left(I_{0}^{i}, \ldots, I_{r}^{i}\right), i \in \mathcal{J}$, with the property that

$$
\left.f_{r}^{i_{1}}\right|_{I_{r}^{i_{1}} \cap I_{r}^{i_{2}}}=\left.f_{r}^{i_{2}}\right|_{I_{r}^{i_{1}} \cap I_{r}^{i_{2}}}
$$

for all $i_{1}, i_{2} \in \mathcal{J}$ such that $I_{r}^{i_{1}} \cap I_{r}^{i_{2}} \neq \emptyset$. Then there exists a unique $f=$ $\left(f_{0}, \ldots, f_{r}\right) \in \mathcal{Q}_{\left(k_{1}, \ldots, k_{r+1}\right)}^{<k_{1}}\left(I_{0}, \ldots, I_{r}\right)$, such that, for all $j \in\{0, \ldots, r\}$,

$$
\left.f_{j}\right|_{I_{j}^{i}}=f_{j}^{i}, i \in \mathcal{J} \text {. }
$$

Proof. By means of induction on $r-j$ we shall prove the existence and uniqueness of $f_{j} \in \mathcal{Q}_{k_{j+1}}^{<k_{1}}\left(I_{j}\right)$ such that $\left.f_{j}\right|_{I_{j}^{i}}=f_{j}^{i}$ for all $i \in \mathcal{J}$. For $j=r$ this statement is true. Now suppose it is true for some $j \leq r$. Then we have

$$
\left.f_{j-1}^{i}\right|_{I_{j}^{i}}=\left.f_{j}\right|_{I_{j}^{i}} \quad\left(\bmod \mathcal{A}^{\leq-k_{j}}\right), \quad i \in \mathcal{J} .
$$

Hence it follows that

$$
\left.\left(f_{j-1}^{i_{2}}-f_{j-1}^{i_{1}}\right)\right|_{I_{j}^{i_{1}} \cap I_{j}^{i_{2}}}=0 \quad\left(\bmod \mathcal{A}^{\leq-k_{j}}\right)
$$

for all $i_{1}, i_{2} \in \mathcal{J}$ such that $I_{r}^{i_{1}} \cap I_{r}^{i_{2}} \neq \emptyset$. In view of Proposition 2.2 this implies that

$$
\left.\left(f_{j-1}^{i_{2}}-f_{j-1}^{i_{1}}\right)\right|_{I_{j-1}^{i_{1}} \cap I_{j-1}^{i_{2}}}=0 \quad\left(\bmod \mathcal{A}^{\leq-k_{j}}\right) .
$$

Consequently, there exists a unique $f_{j-1} \in \mathcal{Q}_{k_{j}}^{<k_{1}}\left(I_{j-1}\right)$ such that $\left.f_{j-1}\right|_{I_{j-1}^{i}}=$ $\left.f_{j-1}^{i}\right|_{I_{j-1}^{i}}$ for $i \in \mathcal{J}$. Thus the statement is true for all $j \in\{0, \ldots, r\}$. This implies that, for all $j \in\{1, \ldots, r\}$, and $i \in \mathcal{J}$,

$$
\left.f_{j-1}\right|_{I_{j}^{i}}=\left.f_{j}\right|_{I_{j}^{i}} \quad\left(\bmod \mathcal{A}^{\leq-k_{j}}\right)
$$


and thus

$$
\left.f_{j-1}\right|_{I_{j}}=f_{j} \quad\left(\bmod \mathcal{A}^{\leq-k_{j}}\right) .
$$

Hence the result follows.

Lemma 2.12. Let $\mathbf{k}:=\left(k_{1}, \ldots, k_{r}\right)$ and let $\mathbf{d}^{1}$ and $\mathbf{d}^{2} \in \mathbf{R}^{r}$ be two multidirections with the property that $\left|d_{j}^{1}-d_{j}^{2}\right|<\frac{\pi}{k_{j}}$ for all $j \in\{1, \ldots, r\}$. Let $\hat{f}$ be $\mathbf{k - s u m m a b l e ~ i n ~ t h e ~ m u l t i - d i r e c t i o n s ~} \mathbf{d}^{1}$ and $\mathbf{d}^{2}$ with multi-sums $\left(f_{1}^{1}, \ldots, f_{r}^{1}\right)$ and $\left(f_{1}^{2}, \ldots, f_{r}^{2}\right)$, respectively. If $f_{r}^{1}=f_{r}^{2}$, then $\hat{f}$ is $\mathbf{k}$ - summable in every multi-direction $\mathbf{d}$ such that $d_{j} \in\left[\min \left\{d_{j}^{1}, d_{j}^{2}\right\}, \max \left\{d_{j}^{1}, d_{j}^{2}\right\}\right]$.

Proof. Let $k_{r+1}=\infty$. By assumption, there exists a positive number $\epsilon$ such that, for $i \in\{1,2\},\left(f_{0}, f_{1}^{i}, \ldots, f_{r}^{i}\right) \in \mathcal{Q}_{\left(k_{1}, \ldots, k_{r+1}\right)}^{\left(k_{1}\right)}\left(I_{0}^{i}, \ldots, I_{r}^{i}\right)$, where $I_{0}^{1}=I_{0}^{2}=\mathbf{R}$ and $I_{j}^{i}=\left(d_{j}^{i}-\epsilon-\frac{\pi}{2 k_{j}}, d_{j}^{i}+\epsilon+\frac{\pi}{2 k_{j}}\right)$ for $j \in\{1, \ldots, r\}$. Let $f_{0}=J^{-1}(\hat{f}), I_{0}=\mathbf{R}$ and, for $j=1, \ldots, r$, let $I_{j}=\left(\min \left\{d_{j}^{1}, d_{j}^{2}\right\}-\frac{\pi}{2 k_{j}}-\right.$ $\left.\epsilon, \max \left\{d_{j}^{1}, d_{j}^{2}\right\}+\frac{\pi}{2 k_{j}}+\epsilon\right)$. Obviously, $I_{j}^{1} \cap I_{j}^{2} \neq \emptyset$ for all $j \in\{1, \ldots, r\}$. Thus, according to Lemma 2.11, there exist quasi-functions $f_{1}, \ldots, f_{r}$ such that $\left(f_{0}, f_{1}, \ldots, f_{r}\right) \in \mathcal{Q}_{\left(k_{1}, \ldots, k_{r+1}\right)}^{<k_{1}}\left(I_{0}, \ldots, I_{r}\right)$.

Lemma 2.13. Let $r \in \mathbf{N}$, let $0<k_{1}<\cdots<k_{r+1}=\infty$. Let $I_{j}^{i}, j=$ $1, \ldots, r, i \in \mathbf{Z}$, be open intervals of $\mathbf{R}$ such that $I_{r}^{i} \subset I_{r-1}^{i} \subset \ldots \subset I_{1}^{i}$ and $\left|I_{j}^{i} \cap I_{j}^{i+1}\right|>\frac{\pi}{k_{j}}$ for all $i \in \mathbf{Z}$. Suppose there exist $f^{i}=\left(f_{1}^{i}, \ldots, f_{r}^{i}\right) \in$ $\mathcal{Q}_{\left(k_{2}, \ldots, k_{r+1}\right)}^{<k_{1}}\left(I_{1}^{i}, \ldots, I_{r}^{i}\right), i \in \mathbf{Z}$, with the property that

$$
\left(\left.f_{1}^{i}\right|_{I_{1}^{i} \cap I_{1}^{i+1}}-\left.f_{1}^{i+1}\right|_{I_{1}^{i} \cap I_{1}^{i+1}}\right) \quad\left(\bmod \mathcal{A}^{\leq-k_{1}}\right)=0
$$

for all $i \in \mathbf{Z}$. Let $I_{j}:=\cup_{i \in \mathbf{Z}} I_{j}^{i}$. Then there exists a unique $f=\left(f_{1}, \ldots, f_{r}\right) \in$ $\mathcal{Q}_{\left(k_{2}, \ldots, k_{r+1}\right)}^{<k_{1}}\left(I_{1}, \ldots, I_{r}\right)$, such that, for all $j \in\{1, \ldots, r\}$,

$$
\left.f_{j}\right|_{I_{j}^{i}}=f_{j}^{i}, i \in \mathbf{Z}
$$

Proof. By means of induction on $j$ one shows that, for all $j \in\{1, \ldots, r\}$ and all $i \in \mathbf{Z}$,

$$
f_{j}^{i}-f_{j}^{i+1} \in \mathcal{Q}_{k_{j+1}}^{\leq-k_{j}}\left(I_{j}^{i} \cap I_{j}^{i+1}\right)
$$

and, consequently, by Proposition 2.3,

$$
\left.f_{j}^{i}\right|_{I_{j}^{i} \cap I_{j}^{i+1}}-\left.f_{j}^{i+1}\right|_{I_{j}^{i} \cap I_{j}^{i+1}}=0 .
$$

Hence the quasifunctions $f_{j}^{i}$ can be glued together to an element of $\mathcal{Q}_{k_{j+1}}^{<k_{1}}\left(\cup_{i \in \mathbf{Z}} I_{j}^{i}\right)$ and the statement of the lemma follows immediately. 
The following lemma is concerned with the Stokes phenomenon, i.e. the difference between two multi-sums of the same formal power series on different, overlapping, multi-intervals. It will be used in $\S 4$ and $\S 5$.

Lemma 2.14. Let $r \in \mathbf{N}, 0<k_{1}<k_{2}<\cdots<k_{r+1}=\infty$. Let $i \in$ $\{1, \ldots, r\}$ and let $\mathbf{I}, \mathbf{I}^{\prime} \in \mathbf{R}^{r}$ be two nested multi-intervals with the property that $I_{j}=I_{j}^{\prime}$ and $\left|I_{j}\right|>\frac{\pi}{k_{j}}$ for all $j \neq i$ and $\left|I_{i} \cap I_{i}^{\prime}\right|=\frac{\pi}{k_{i}}$. Suppose that there exist $\mathbf{f}=\left(f_{1}, \ldots, f_{r}\right) \in \mathcal{Q}_{\left(k_{2}, \ldots, k_{r+1}\right)}^{<k_{1}}(\mathbf{I})$ and $\mathbf{f}^{\prime}=\left(f_{1}^{\prime}, \ldots, f_{r}^{\prime}\right) \in$ $\mathcal{Q}_{\left(k_{2}, \ldots, k_{r+1}\right)}^{<k_{1}}\left(\mathbf{I}^{\prime}\right)$, such that $\left(f_{1}^{\prime}-f_{1}\right)\left(\bmod \mathcal{A}^{\leq-k_{1}}\right)=0$. Then $f_{j}^{\prime}=f_{j}$ for all $j<i$, whereas

$$
f_{i}^{\prime}-f_{i} \in \mathcal{Q}_{k_{i+1}}^{\leq-k_{i}}\left(\left(-\frac{\pi}{2 k_{i}}, \frac{\pi}{2 k_{i}}\right)\right)
$$

and, for all $j>i$,

$$
f_{j}^{\prime}-f_{j} \in \mathcal{Q}_{k_{j+1}}^{\leq-k_{i}}\left(I_{j}\right)
$$

Proof. For all $j \in\{1, \ldots, r\}$ we have

$$
f_{j}^{\prime}-f_{j} \in \mathcal{Q}_{k_{j+1}}^{<k_{1}}\left(I_{j} \cap I_{j}^{\prime}\right)
$$

and, for all $j>1$,

$$
\left(f_{j}^{\prime}-f_{j}\right) \quad\left(\bmod \mathcal{A}^{\leq-k_{j}}\right)=\left.\left(f_{j-1}^{\prime}-f_{j-1}\right)\right|_{I_{j} \cap I_{j}^{\prime}} .
$$

By means of induction on $j$ and Proposition 2.3 it is shown that, for all $j \leq i$,

$$
f_{j}^{\prime}-f_{j} \in \mathcal{Q}_{k_{j+1}}^{\leq-k_{j}}\left(I_{j} \cap I_{j}^{\prime}\right)
$$

and $f_{j}^{\prime}=f_{j}$ for all $j<i$, and $f_{i}^{\prime}-f_{i} \in \mathcal{Q}_{k_{i+1}}^{\leq-k_{i}}\left(I_{i} \cap I_{i}^{\prime}\right)$. The remaining part of the lemma now follows easily.

In various applications (e.g. in the theory of linear differential equations, cf. §1) one deals with multi-summable formal power series having a finite number of singular directions of each level in any finite interval. In that case, each quasi-function in a multi-sum of the formal series possesses a finite representative, consisting of multi-sums of the same formal power series.

Lemma 2.15. Let $r \in \mathbf{N}, 0<k_{1}<k_{2}<\cdots<k_{r+1}=\infty$. Let $\hat{f} \in \mathbf{C}[[z]]_{k_{1}}$ and suppose that $\hat{f}$ possesses a $\left(k_{1}, \ldots, k_{r}\right)$-sum $\left(f_{1}, \ldots, f_{r}\right)$ on the multiinterval $\left(I_{1}, \ldots, I_{r}\right)$ where $I_{1}, \ldots, I_{r}$ are open. Assume that the interval $I_{1}$ only contains a finite number of Stokes pairs of level $k_{j}$ for each $j \in$ $\{2, \ldots, r\}$. Then the quasi-function $f_{j}$ has a finite representative of the form $\left\{f^{(\nu)}: \nu \in\left\{M_{j}, \ldots, N_{j}\right\}\right\}$, where $M_{1} \leq M_{2} \leq \cdots \leq M_{r} \leq N_{r} \leq \cdots \leq$ $N_{2} \leq N_{1}$ and $f^{(\nu)}$ is a $\left(k_{1}, \ldots, k_{r}\right)$-sum of $\hat{f}$ for each $\nu \in\left\{M_{1}, \ldots, N_{1}\right\}$. 
Proof. There exists a covering of $I_{1}$ by intervals $I^{(\nu)}=\left(a^{(\nu)}, b^{(\nu)}\right), \nu=$ $M_{1}, \ldots, N_{1}$, where $M_{1}$ and $N_{1}$ are integers with $M_{1}<N_{1}$, such that $\left|I^{(\nu)}\right|>$ $\pi / k_{r}, I^{(\nu)}$ does not contain any Stokes pair of level $k_{j}$ for $j=2, \ldots, r$, $a^{(\nu)}<a^{(\nu+1)}<b^{(\nu)}<b^{(\nu+1)}$, and for all $j \in\{1, \ldots, r\}$ there exist integers $M_{j}$ and $N_{j}$ such that $I_{j}=\left(a^{\left(M_{j}\right)}, b^{\left(N_{j}\right)}\right)$. Hence $M_{1} \leq M_{2} \leq \cdots \leq M_{r} \leq$ $N_{r} \leq \cdots \leq N_{2} \leq N_{1}$. We may assume that $M_{r}=N_{r}$.

Let $\nu \in\left\{M_{1}, \ldots, N_{1}\right\}$ and let $j$ be the largest integer such that $I^{(\nu)} \subset I_{j}$. Then we define $I_{h}^{(\nu)}:=I_{h}$ if $h \in\{1, \ldots, j\}$ and $I_{r}^{(\nu)}:=I^{(\nu)}$. If $j<r-1$, there exist open intervals $I_{h}^{(\nu)}$ of length $>\pi / k_{h}, h \in\{j+1, \ldots, r-1\}$, such that $I_{h}^{(\nu)}$ does not contain any Stokes pair of level $k_{h}$ and $\left(I_{1}^{(\nu)}, \ldots, I_{r}^{\nu}\right)$ is a nested multi-interval. Then, by Corollary 2.10, $\hat{f}$ is multi-summable on this multi-interval with multisum $\left(f_{1}^{(\nu)}, \ldots, f_{r}^{(\nu)}\right)$. Note that $I_{1}^{\nu}=I_{1}$ and $f_{1}^{\nu}=f_{1}$. Let $f^{(\nu)}:=f_{r}^{(\nu)}$.

If $M_{j} \leq \nu \leq N_{j}$ and $h \in\{1, \ldots, j\}$, then $I_{h}^{(\nu)}=I_{h}$ and from Proposition 2.3 it then follows that $f_{h}^{(\nu)}=f_{h}$. Furthermore, $\left.f_{j}\right|_{I^{(\nu)}}=\left.f_{j}^{(\nu)}\right|_{I^{(\nu)}}=f^{(\nu)}$ $\left(\bmod \mathcal{A}^{\leq-k_{j+1}}\right)$ which implies that $f_{j}$ can be represented by $\left\{f^{(\nu)}, \nu=\right.$ $\left.M_{j}, \ldots, N_{j}\right\}$.

\section{Multisummability and Cauchy-Heine transforms.}

Suppose we are given two open intervals $I_{1}$ and $I_{2}$ such that $I_{1} \cap I_{2} \neq \emptyset$, and two analytic functions $f_{1} \in \mathcal{A}^{(0)}\left(I_{1}\right)$ and $f_{2} \in \mathcal{A}^{(0)}\left(I_{2}\right)$, admitting the same asymptotic expansion $\hat{f}$ as $z \rightarrow 0$. Obviously, the difference $f_{1}-f_{2}$ belongs to $\mathcal{A}^{<0}\left(I_{1} \cap I_{2}\right)$. Conversely, if we are given an interval $I=(\alpha, \beta)$ and a function $f \in \mathcal{A}^{<0}(I)$, we can construct two functions $f_{1} \in \mathcal{A}^{(0)}((\alpha, \beta+2 \pi))$ and $f_{2} \in \mathcal{A}^{(0)}((\alpha-2 \pi, \beta))$ with the property that $\hat{f}_{1}=\hat{f}_{2}$ and $f_{1}-f_{2}=f$. To this end, choose $\rho \in \mathbf{C}$ such that $\arg \rho \in I$ and $f$ is holomorphic on $(0, \rho)$. Now define $f_{1}$ and $f_{2}$ by

$$
f_{1}(z):=C H_{\rho}^{-}(f):=\int_{0}^{\rho} \frac{f(\zeta)}{2 \pi i(\zeta-z)} d \zeta \quad-2 \pi<\arg z-\arg \rho<0
$$

and

$$
f_{2}(z):=C H_{\rho}^{+}(f):=\int_{0}^{\rho} \frac{f(\zeta)}{2 \pi i(\zeta-z)} d \zeta \quad 0<\arg z-\arg \rho<2 \pi .
$$

$C H_{\rho}^{-}(f)$ and $C H_{\rho}^{+}(f)$ are so-called Cauchy-Heine transforms of $f$. Note that $C H_{\rho}^{-}(f)(z)=C H_{\rho}^{+}(f)\left(z e^{2 \pi i}\right)$. By deformation of the path of integration, the functions $f_{1}$ and $f_{2}$ can be continued analytically to elements of $\mathcal{A}((\alpha-$ $2 \pi, \beta))$ and $\mathcal{A}((\alpha, \beta+2 \pi))$, respectively. With the aid of the expansion $\sum_{n=0}^{\infty} \zeta^{-n-1} z^{n}$ for $(\zeta-z)^{-1}$, it can be shown that $f_{1}$ and $f_{2}$ admit an 
asymptotic expansion $\widehat{C H}_{\rho}(f)=\sum_{n=0}^{\infty} a_{n} z^{n}$ as $z \rightarrow 0$, where

$$
a_{n}=\frac{1}{2 \pi i} \int_{0}^{\rho} f(\zeta) \zeta^{-n-1} d \zeta
$$

It is easily seen that $f_{1}-f_{2}=f$. A different choice of $\rho$ leads to a pair of functions $\tilde{f}_{1}$ and $\tilde{f}_{2}$ such that $\tilde{f}_{1}-f_{1}=\tilde{f}_{2}-f_{2}$ and this difference is regular at $O$. The construction described above is extremely useful in many applications. For example, suppose we are given a formal power series $\hat{f} \in \mathbf{C}[[z]]$. $\hat{f}$ defines an element $f_{0} \in \mathcal{A}^{(0)} / \mathcal{A}^{<0}\left(S^{1}\right)$, where $S^{1}$ is the unit circle and, with a slight abuse of notation, we let $\mathcal{A}^{(0)}$ and $\mathcal{A}^{<0}$ denote sheaves on $S^{1}$ instead of $\mathbf{R}$. $f_{0}$ can be represented by a 0 -cochain of $\mathcal{A}^{(0)}$ with respect to some open covering $\left\{U_{i}\right\}_{i=1}^{N}$ of $S^{1}$ and its coboundary defines an element of $H^{1}\left(S^{1}, \mathcal{A}^{<0}\right)$, which is independent of the choice of representative of $f_{0}$. Conversely, if $\left\{g_{i}\right\}_{i=1}^{M}$ represents an element $\Phi$ of $H^{1}\left(S^{1}, \mathcal{A}^{<0}\right)$, then, with the aid of Cauchy-Heine transforms of the $g_{i}$, we can construct a 0 -cochain $\left\{f_{i}\right\}_{i=1}^{N}$ with coboundary $\left\{g_{i}\right\}_{i=1}^{M}$ and asymptotic expansion $\hat{f}_{i}=\hat{f} \in \mathbf{C}[[z]]$ for all $i \in\{1, \ldots, N\}$. The equivalence class modulo $\mathbf{C}\{z\}$ of the formal power series $\hat{f}$ is independent of the choice of cochain representing $\Phi$. This construction yields a well-known isomorphism from $H^{1}\left(S^{1}, \mathcal{A}^{<0}\right)$ onto $\mathbf{C}[[z]] / \mathbf{C}\{z\}$. In an analogous manner one can construct an isomorphism from $H^{1}\left(S^{1}, \mathcal{A}^{\leq-k}\right)$ onto $\mathbf{C}[[z]]_{k} / \mathbf{C}\{z\}$, for any positive number $k$ (cf. $[\mathbf{1 8}, \mathbf{2 3}]$ ). In this section we derive similar results for multi-summable power series. To that end, we begin by studying Cauchy-Heine transforms of functions with properties that are characteristic of differences of multi-sums.

In this and the next section, we shall frequently use the symbols \pm and F to combine two statements: One statement is obtained by reading the upper signs, the other one by reading the lower signs.

Definition 3.1. Let $f \in \mathcal{A}^{<0}(I)$ where $I$ is an open interval of $\mathbf{R}$. By $C H^{ \pm}(f)$ we denote the equivalence class modulo $\mathbf{C}\{z\}$ of the function defined by the (analytic continuation of the) Cauchy-Heine transform

$$
C H_{\rho}^{ \pm}(f):=\int_{0}^{\rho} \frac{f(\zeta)}{2 \pi i(\zeta-z)} d \zeta \quad 0< \pm(\arg z-\arg \rho)<2 \pi
$$

where $|\rho|$ is sufficiently small and $\arg \rho \in I$.

By $\widehat{C H}(f)$ we denote the equivalence class modulo $\mathbf{C}\{z\}$ of the formal power series expansion of the function defined above.

Lemma 3.2 (cf. Ramis, [23]). Let $k>0$ and let $a, b \in \mathbf{R}: a<b$. If $f \in \mathcal{A}^{\leq-k}((a, b))$ then

$$
C H^{+}(f) \in \mathcal{A}^{(k)}((a, b+2 \pi)) / \mathbf{C}\{z\}
$$

and

$$
C H^{-}(f) \in \mathcal{A}^{(k)}((a-2 \pi, b)) / \mathbf{C}\{z\} .
$$


For a proof of this lemma cf. [19, pp. 114, 115].

Corollary 3.3. Let $k>0$, let $a, b$ be real numbers such that $a<b$, and $f \in \mathcal{A}^{\leq-k}((a, b))$. Then $F:=\widehat{C H}(f) \in \mathbf{C}\{z\}_{\mathbf{k}, \mathbf{d}} / \mathbf{C}\{z\}$ for all $d \in(a-$ $\left.2 \pi+\frac{\pi}{2 k}, b-\frac{\pi}{2 k}\right) \bmod 2 \pi$. CH $H^{+}(f)$ is the k-sum of $F$ in the directions $d \in\left(a+\frac{\pi}{2 k}, b+2 \pi-\frac{\pi}{2 k}\right)$ and $C H^{-}(f)$ is the $k$-sum of $F$ in the directions $d \in\left(a-2 \pi+\frac{\pi}{2 k}, b-\frac{\pi}{2 k}\right)$. In particular, if $a=-\frac{\pi}{2 k}$ and $a<b+2 \pi-\frac{\pi}{k}$, then $\mathrm{CH}^{+}(f)$ is a k-sum in the directions $d \in(0, \epsilon)$ for some $\epsilon>0$, and if $a<-\frac{\pi}{2 k}$ then it is a $k$-sum in the direction 0 . Similarly, if $b=\frac{\pi}{2 k}$ and $a<b+2 \pi-\frac{\pi}{k}$, then $\mathrm{CH}^{-}(f)$ is a $k$-sum in the directions $d \in(-\epsilon, 0)$ for some $\epsilon>0$, and if $b>\frac{\pi}{2 k}$ it is a $k$-sum in the direction 0 .

Lemma 3.4. Let $I_{0}=\left(a_{0}, b_{0}\right) \subset I=(a, b), 0<k<l, f_{0} \in \mathcal{A}^{\leq-k}\left(I_{0}\right), f \in$ $\mathcal{A}^{\leq-k} / \mathcal{A}^{\leq-l}(I)$, such that $\left.f\right|_{I_{0}}=f_{0}\left(\bmod \mathcal{A}^{\leq-l}\right)$. Then there exists $F \in$ $\mathcal{A}^{(k)} / \mathcal{A} \leq-l(a, b+2 \pi)$ with the property that

$$
\left.F\right|_{\left(a_{0}, b_{0}+2 \pi\right)} \quad(\bmod \mathbf{C}\{z\})=C H^{+}\left(f_{0}\right) \quad\left(\bmod \mathcal{A}^{\leq-l}\right) .
$$

If there exist $b^{+} \in(a, b), b^{+}<b_{0}+2 \pi$ and $f^{+} \in \mathcal{A}^{\leq-k}\left(b^{+}, b\right)$ such that $\left.f\right|_{\left(b^{+}, b\right)}=f^{+}\left(\bmod \mathcal{A}^{\leq-l}\right)$ then there exists $g^{+} \in \mathcal{A}^{(l)}\left(b^{+}, b_{0}+2 \pi\right)$ with the property that

$$
\left.C H^{+}\left(f_{0}\right)\right|_{\left(b^{+}, b_{0}+2 \pi\right)}=\left.C H^{+}\left(f^{+}\right)\right|_{\left(b^{+}, b_{0}+2 \pi\right)}+g^{+} \quad(\bmod \mathbf{C}\{z\}) .
$$

Similarly, if there exist $a^{-} \in(a, b), a^{-}>a_{0}-2 \pi$ and $f^{-} \in \mathcal{A}^{\leq-k}\left(a, a^{-}\right)$ such that $\left.f\right|_{\left(a, a^{-}\right)}=f^{-}\left(\bmod \mathcal{A}^{\leq-l}\right)$ then there exists $g^{-} \in \mathcal{A}^{(l)}\left(a_{0}, a^{-}+2 \pi\right)$ with the property that

$$
\left.C H^{+}\left(f_{0}\right)\right|_{\left(a_{0}, a^{-}+2 \pi\right)}=\left.C H^{+}\left(f^{-}\right)\right|_{\left(a_{0}, a^{-}+2 \pi\right)}+g^{-} \quad(\bmod \mathbf{C}\{z\}) .
$$

Proof. Let $f$ be represented by $\left\{f_{j}\right\}_{j \in \mathcal{J}}$ on a covering $\left\{I_{j}\right\}_{j \in \mathcal{J}}$ of $I$ where $\mathcal{J}$ is an interval of $\mathbf{Z}$ containing 0 , and $I_{j}=\left(a_{j}, b_{j}\right)$ with $a_{j}<a_{j+1}<b_{j}<b_{j+1}$. Thus $f_{j} \in \mathcal{A}^{\leq-k}\left(I_{j}\right), f_{j}-f_{j+1} \in \mathcal{A}^{\leq-l}\left(I_{j} \cap I_{j+1}\right)$.

According to Lemma $3.2, \mathrm{CH}^{+}\left(f_{j}\right)$ possesses a representative $\phi_{j} \in$ $\mathcal{A}^{(k)}\left(a_{j}, b_{j}+2 \pi\right)$. Let $\psi_{j}:=\phi_{j}-\phi_{j+1}$. Then, again by Lemma 3.2 , $\psi_{j} \in \mathcal{A}^{(l)}\left(a_{j+1}, b_{j}+2 \pi\right)$ with an asymptotic expansion $\hat{\psi}_{j} \in \mathbf{C}[[z]]_{l}$.

Let $N \geq 0, N \in \mathcal{J}$ and define $\rho_{N}:=\sum_{j=0}^{N-1} J^{-1} \hat{\psi}_{j}$ (cf. Lemma 2.5). Thus $\rho_{N} \in \mathcal{A}^{(l)} / \mathcal{A}^{\leq-l}(\mathbf{R})$. If $a_{N}<b_{0}+2 \pi$ we define $\chi_{N}=\sum_{j=0}^{N-1} \psi_{j}$, so that

$$
\chi_{N} \in \mathcal{A}^{(l)}\left(a_{N}, b_{0}+2 \pi\right) \text { and }\left.\phi_{0}\right|_{\left(a_{N}, b_{0}+2 \pi\right)}=\left.\phi_{N}\right|_{\left(a_{N}, b_{0}+2 \pi\right)}+\chi_{N}
$$

and

$$
\left.\rho_{N}\right|_{\left(a_{N}, b_{0}+2 \pi\right)}=\chi_{N} \quad\left(\bmod \mathcal{A}^{\leq-l}\right) .
$$

Define

$$
F_{N}=\left(\phi_{N}+\left.\rho_{N}\right|_{\left(a_{N}, b_{N}+2 \pi\right)}\right) \quad\left(\bmod \mathcal{A}^{\leq-l}\right) .
$$


Then $F_{N} \in \mathcal{A}^{(k)} / \mathcal{A}^{\leq-l}\left(a_{N}, b_{N}+2 \pi\right)$ and if $a_{N}<b_{0}+2 \pi$ then $\left.F_{N}\right|_{\left(a_{N}, b_{0}+2 \pi\right)}=$ $\left.\phi_{0}\right|_{\left(a_{N}, b_{0}+2 \pi\right)}\left(\bmod \mathcal{A}^{\leq-l}\right)$.

Assume that also $N+1 \in \mathcal{J}$. Let $\rho_{N}$ be represented by $\tilde{\rho}_{N} \in \mathcal{A}^{(l)}(\tilde{I})$ on some interval $\tilde{I} \subset\left(a_{N+1}, b_{N}+2 \pi\right)$. Then $F_{N}$ may be represented on $\tilde{I}$ by $\phi_{N}+\tilde{\rho}_{N}=\phi_{N+1}+\tilde{\rho}_{N+1}$ where $\tilde{\rho}_{N+1}:=\psi_{N}+\tilde{\rho}_{N}$, and so $\tilde{\rho}_{N+1}$ is a representative of $\hat{\rho}_{N+1}$ on $\tilde{I}$. Hence $F_{N}$ and $F_{N+1}$ have a representative in common and so by quasi-analyticity $\left.F_{N}\right|_{\left(a_{N+1}, b_{N}+2 \pi\right)}=\left.F_{N+1}\right|_{\left(a_{N+1}, b_{N}+2 \pi\right)}$. The sheaf property now implies that the functions $F_{N}$ with $N \geq 0$ determine an element $F \in \mathcal{A}^{(k)} /\left(\mathcal{A}^{\leq-l}\left(a_{0}, b+2 \pi\right)\right)$ such that (20) holds.

In a similar way we may extend $\phi_{0}$ to quasi-functions on intervals which start to the left of $a_{0}$ by choosing $N \leq 0, N \in \mathcal{J}$ and

$$
\chi_{N}=-\sum_{j=N}^{-1} \psi_{j} \text { if } a_{0}<b_{N}+2 \pi \text { and } \rho_{N}:=\sum_{j=N}^{-1} \hat{\psi}_{j} .
$$

The corresponding $F_{N}$ with $N<0$ can be pasted with those with $N \geq 0$ to obtain $F \in \mathcal{A}^{(k)} / \mathcal{A}^{\leq-l}(a, b+2 \pi)$.

If the condition on $f^{+}$is fulfilled then we may choose $N$ such that $a_{N}=$ $b^{+}, b_{N}=b, f_{N}=f^{+}, \phi_{N}$ is a representative of $C H^{+}\left(f^{+}\right)$and thus we obtain (21) from (23) with $g^{+}:=\chi_{N}$. Similarly if the condition on $f^{-}$is fulfilled.

From this lemma we will derive:

Corollary 3.5. Let $r \in \mathbf{N}, 0<k_{1}<\cdots<k_{r}<k_{r+1}=\infty$, I $:=\left(I_{1}, \ldots, I_{r}\right)$ be a nested multi-interval with $I_{j}=\left(a_{j}, b_{j}\right), j=1, \ldots, r$ and $\mathbf{f}:=\left(f_{1}, \ldots, f_{r}\right)$ $\in \mathcal{Q}_{\left(k_{2}, \ldots, k_{r+1}\right)}^{\leq-k_{1}}(\mathbf{I})$. Let $\tilde{I}_{j}:=\left(a_{j}, b_{j}+2 \pi\right)$ and $\tilde{\mathbf{I}}:=\left(\tilde{I}_{1}, \ldots, \tilde{I}_{r}\right)$.

Then there exists $\mathbf{F}=\left(F_{1}, \ldots, F_{r}\right) \in \mathcal{Q}_{\left(k_{2}, \ldots, k_{r+1}\right)}^{\left(k_{1}\right)}(\tilde{\mathbf{I}})$ such that $F_{r}(\bmod \mathbf{C}\{z\})=C H^{+}\left(f_{r}\right)$. If $\left|\tilde{I}_{j}\right|>\pi / k_{j}$ for $j=1, \ldots, r-$ so in particular if $k_{1} \geq 1 / 2$ - then $\mathbf{F}$ is the $\left(k_{1}, \ldots, k_{r}\right)$-sum of $\hat{F}_{0}$ on $\tilde{\mathbf{I}}$ where $\hat{F}_{0}$ $(\bmod \mathbf{C}\{z\})=\widehat{C H}\left(f_{r}\right)$.

If, in addition, for all $j \in\{1, \ldots, r-1\}$ there exist $b_{j}^{+} \in\left(a_{j}, b_{j}\right)$ and $f_{j}^{+} \in \mathcal{A}^{\leq-k_{1}}\left(b_{j}^{+}, b_{j}\right)$ such that $b_{j}^{+}<b_{r}+2 \pi$ and $f_{j}$ is represented by $f_{j}^{+}$ on $\left(b_{j}^{+}, b_{j}\right)$ then for $j=1, \ldots, r$ there exists $g_{j}^{+} \in \mathcal{A}^{\left(k_{j}\right)}\left(b_{j-1}^{+}, b_{j}+2 \pi\right)$ with $b_{0}^{+}:=b_{1}^{+}$such that

$$
C H^{+}\left(f_{r}\right)=\sum_{j=1}^{r} g_{j}^{+} \quad(\bmod \mathbf{C}\{z\})
$$

where in both sides we take the restriction to the intersection of the intervals on which the terms are well-defined.

Similarly, if for all $j \in\{1, \ldots, r-1\}$ there exists $a_{j}^{-} \in\left(a_{j}, b_{j}\right)$ such that $a_{r}<a_{j}^{-}+2 \pi$ and $f_{j}$ is represented by a function on $\left(a_{j}, a_{j}^{-}\right)$then for 
$j=1, \ldots, r$ there exists $g_{j}^{-} \in \mathcal{A}^{\left(k_{j}\right)}\left(a_{j}, a_{j-1}^{-}+2 \pi\right]$ with $a_{0}^{-}:=a_{1}^{-}$such that

$$
C H^{+}\left(f_{r}\right)=\sum_{j=1}^{r} g_{j}^{-} \quad(\bmod \mathbf{C}\{z\})
$$

where in both sides we take the restriction to the intersection of the intervals on which the terms are well-defined.

Proof. We may apply the previous lemma with $I_{0}:=I_{r}, f_{0}:=f_{r}, k:=$ $k_{1}, f:=f_{j}, l:=k_{j+1}, I:=I_{j}$. Hence there exist $F_{j} \in \mathcal{A}^{\left(k_{1}\right)} / \mathcal{A}^{\leq-k_{j+1}}\left(\tilde{I}_{j}\right)$ such that

$$
\left.F_{j}\right|_{\tilde{I}_{r}}=\phi_{r} \quad\left(\bmod \mathcal{A}^{\leq-k_{j+1}}\right),
$$

where $\phi_{r}$ is a representative of $C H^{+}\left(f_{r}\right)$. Hence $\left.F_{j-1}\right|_{\tilde{I}_{r}}=\left.F_{j}\left(\bmod \mathcal{A}^{\leq-k_{j}}\right)\right|_{\tilde{I}_{r}}$. With the aid of Proposition 2.2 we now deduce the first assertion.

Next let the assumptions concerning $(24)$ be satisfied. Let $\left.f_{j}\right|_{\left(b_{j}^{+}, b_{j}\right)}$ be represented by $f_{j}^{+} \in \mathcal{A}^{\leq-k_{1}}\left(b_{j}^{+}, b_{j}\right), j=1, \ldots, r-1$. Let $f_{r}^{+}:=f_{r}, b_{r}^{+}:=$ $a_{r}$. Then we may apply the preceding lemma with $k:=k_{1}, l:=k_{j}, f_{0}:=$ $f_{j}^{+}, I_{0}:=\left(b_{j}^{+}, b_{j}\right), f:=f_{j-1}, I:=I_{j-1}, f^{+}:=f_{j-1}^{+}, b^{+}:=b_{j-1}^{+}$where $j=$ $2, \ldots, r$. It follows that there exists $g_{j}^{+} \in \mathcal{A}^{\left(k_{j}\right)}\left(b_{j-1}^{+}, b_{j}+2 \pi\right)$ such that

$$
\begin{aligned}
& \left.C H^{+}\left(f_{j}\right)^{+}\right|_{\left(b_{j-1}^{+}, b_{j}+2 \pi\right)} \\
& =g_{j}^{+}+\left.C H^{+}\left(f_{j-1}^{+}\right)\right|_{\left(b_{j-1}^{+}, b_{j}+2 \pi\right)} \quad(\bmod \mathbf{C}\{z\}), j=2, \ldots, r .
\end{aligned}
$$

With $g_{1}^{+}:=C H^{+}\left(f_{1}^{+}\right) \in \mathcal{A}^{\left(k_{1}\right)}\left(b_{1}^{+}, b_{1}+2 \pi\right)$ (cf. Lemma 3.2) we obtain (24) by addition. The proof of (25) is similar.

Remark 3.6. Similar results hold for $\mathrm{CH}^{-}$since $\mathrm{CH}^{-}(f)(z)=$ $C H^{+}(f)\left(z e^{2 \pi i}\right)$. The corollary may be applied to the case that $\mathbf{f}=h \mathbf{g}$ where $\mathbf{g} \in \mathcal{Q}_{\left(k_{2}, \ldots, k_{r+1}\right)}^{<k_{1}}(\mathbf{I})$ and $h \in \mathcal{A}^{\leq-k_{1}}\left(I_{1}\right)$.

The above corollary is a generalization of Balser: [1, $\S 6.7$, Proposition 4].

According to a well-known result, due to B. Malgrange and Y. Sibuya (cf. [23]), the set $\mathbf{C}[[z]]_{k} / \mathbf{C}\{z\}$ is isomorphic to $H^{1}\left(S^{1}, \mathcal{A}^{\leq-k}\right)$, where $S^{1}$ denotes the unit circle. In the next two theorems we consider the restriction of this isomorphism to the set of multi-summable power series and give a characterization of its image in $H^{1}\left(S^{1}, \mathcal{A} \leq-k\right)$.

Theorem 3.7. Let $r \in \mathbf{N}, 0<k_{1}<\ldots<k_{r+1}=\infty$. Suppose we are given admissible multi-intervals $\mathbf{I}^{i}=\left(I_{1}^{i}, \ldots, I_{r}^{i}\right)$, where $I_{j}^{i}=\left(\alpha_{j}^{i}, \beta_{j}^{i}\right), j \in$ $\{1, \ldots, r\}, i \in\{1, \ldots, N+1\}$, with the following properties

- $\left|I_{j}^{i}\right|>\frac{\pi}{k_{j}}$,

- $\left\{I_{r}^{i}\right\}_{i=1}^{N}$ is a covering of $[0,2 \pi)$, 
- $\alpha_{j}^{i}<\beta_{j}^{i+1}<\alpha_{j}^{i+1}<\beta_{j}^{i}<\beta_{j}^{N+1}$ for all $i \in\{1, \ldots, N\}$ and $j \in$ $\{1, \ldots, r\}$,

- $\left|I_{1}^{i+1} \cap I_{1}^{i}\right|<2 \pi$ for all $i \in\{1, \ldots, N\}$.

Let $I_{j}^{N+1}=I_{j}^{1}+2 \pi$. Suppose there exist $\mathbf{g}^{i} \in \mathcal{Q}_{\left(k_{2}, \ldots, k_{r+1}\right)}^{\leq-k_{1}}\left(\mathbf{I}^{i+1} \cap \mathbf{I}^{i}\right), i \in$ $\{1, \ldots, N\}$. Then there exists a unique $\hat{F} \in \mathbf{C}[[z]]_{k_{1}},\left(k_{1}, \ldots, k_{r}\right)$-summable on $\mathbf{I}^{i}$ with sum $\mathbf{f}^{i}$ for all $i \in\{1, \ldots, N+1\}$, such that $f_{r}^{N+1}(z)=f_{r}^{1}\left(z e^{-2 \pi i}\right)$ and $f_{r}^{i+1}-f_{r}^{i}=g_{r}^{i}$ for all $i \in\{1, \ldots, N\}$. Moreover, $\hat{F}(\bmod \mathbf{C}\{z\})=$ $\sum_{i=1}^{N} \widehat{C H}\left(g_{r}^{i}\right)$.

Proof. For $i \in\{1, \ldots, N+1\}$, let $f_{r}^{i}=\sum_{h=1}^{N} C H^{d_{i h}}\left(g_{r}^{h}\right)$, where $d_{i h}=+$ if $h<i$ and $d_{i h}=-$ if $h \geq i$. From Corollary 3.5 we deduce that $f_{r}^{i}$ is a $\left(k_{1}, \ldots, k_{r}\right)$-sum on $\left(I_{1}^{i}, \ldots, I_{r}^{i}\right)$. Moreover, $f_{r}^{i+1}-f_{r}^{i}=C H^{+}\left(g_{r}^{i}\right)-$ $\mathrm{CH}^{-}\left(g_{r}^{i}\right)=g_{r}^{i}$ and

$$
f_{r}^{N+1}(z)=\sum_{h=1}^{N} C H^{+}\left(g_{r}^{h}\right)(z)=\sum_{h=1}^{N} C H^{-}\left(g_{r}^{h}\right)\left(z e^{-2 \pi i}\right)=f_{r}^{1}\left(z e^{-2 \pi i}\right) .
$$

Theorem 3.8. Let $\frac{1}{2}<k_{1}<\cdots<k_{r}, k_{r+1}=\infty$ and $\hat{f} \in \mathbf{C}[[z]] . \hat{f} \in$ $\mathbf{C}\{z\}_{\left(k_{1}, \ldots, k_{r}\right)}$ if and only if there exist $d_{i} \in[0,2 \pi), f_{r}^{i} \in \mathcal{A}\left(\left(d_{i}-\frac{\pi}{2 k_{r}}, d_{i+1}+\right.\right.$ $\left.\left.\frac{\pi}{2 k_{r}}\right)\right), i=1, \ldots, N$, where $d_{N+1}:=d_{1}+2 \pi$, and $g_{j}^{i} \in \mathcal{Q}_{k_{j+1}}^{\leq-k_{1}}\left(\left(d_{i+1}-\right.\right.$ $\left.\left.\frac{\pi}{2 k_{j}}, d_{i+1}+\frac{\pi}{2 k_{j}}\right)\right), j=1, \ldots, r, i=1, \ldots, N$, with the following properties:

$f_{r}^{i}(z) \sim \hat{f}(z)$ as $z \rightarrow 0, \arg z \in\left(d_{i}-\frac{\pi}{2 k_{r}}, d_{i+1}+\frac{\pi}{2 k_{r}}\right), i=1, \ldots, N$, $g_{j}^{i}\left(\bmod \mathcal{A}^{\leq-k_{j}}\right)=\left.g_{j-1}^{i}\right|_{\left(d_{i+1}-\frac{\pi}{2 k_{j-1}}, d_{i+1}+\frac{\pi}{2 k_{j-1}}\right)}, j=2, \ldots, r, i=1, \ldots, N$, $f_{r}^{i+1}-f_{r}^{i}=g_{r}^{i}$, for all $i \in\{1, \ldots, N\}$, where $f_{r}^{N+1}$ is defined by $f_{r}^{N+1}(z)=f_{r}^{1}\left(z e^{-2 \pi i}\right)$.

\section{The Stokes phenomenon in the direction 0 .}

In the two remaining sections we shall restrict ourselves to the study of $\left(k_{1}, \ldots, k_{r}\right)$-sums on multi-intervals $\left(I_{1}, \ldots, I_{r}\right)$, where $I_{j}=\left[-\frac{\pi}{2 k_{j}}, \frac{\pi}{2 k_{j}}\right)$, or $I_{j}=\left(-\frac{\pi}{2 k_{j}}, \frac{\pi}{2 k_{j}}\right]$, or $I_{j}=\left[-\frac{\pi}{2 k_{j}}, \frac{\pi}{2 k_{j}}\right]$. Therefore, we introduce the following conventions. If $r \in \mathbf{N}$ and $0<k_{1}<\cdots<k_{r}$ are given, we write

$$
\begin{aligned}
I_{j}^{0}=\left[-\frac{\pi}{2 k_{j}}, \frac{\pi}{2 k_{j}}\right], \quad I_{j}^{+}= & \left(-\frac{\pi}{2 k_{j}}, \frac{\pi}{2 k_{j}}\right] \\
& \text { and } I_{j}^{-}=\left[-\frac{\pi}{2 k_{j}}, \frac{\pi}{2 k_{j}}\right), \quad j \in\{1, \ldots, r\} .
\end{aligned}
$$


We say that $\left(f_{1}, \ldots, f_{r}\right)$ is a $\left(k_{1}, \ldots, k_{r}\right)$-sum in the multi-direction $\left(d_{1}, \ldots\right.$, $\left.d_{r}\right) \in\{+, 0,-\}^{r}$ if $\left(f_{1}, \ldots, f_{r}\right)$ is a $\left(k_{1}, \ldots, k_{r}\right)$-sum on $\left(I_{1}, \ldots, I_{r}\right)$, where $I_{j}=I_{j}^{d_{j}}$.

Definition 4.1. By $\Sigma^{ \pm}$we denote the differential algebra of all multi-sums in the direction \pm , i.e. the set of $f \in \mathcal{A}_{0}$ ( $\mathcal{A}_{0}$ denotes the stalk in $O$ of the sheaf $\mathcal{A}$ ) with the following property: There exist a positive integer $r$, positive numbers $k_{1}, \ldots, k_{r}$ such that $k_{1}<\cdots<k_{r}<k_{r+1}=\infty$ and $\left(f_{1}, \ldots, f_{r}\right) \in \Sigma_{\left(k_{1}, \ldots, k_{r}\right)}\left(I_{1}^{ \pm}, \ldots, I_{r}^{ \pm}\right)$such that $f_{r}=f$.

By $\Sigma^{0}$ we denote the differential algebra of all multi-sums in the direction 0 .

By $\Sigma^{<0}$ we denote the differential algebra of $f \in \mathcal{A}_{0}$ with the following property: There exist a positive integer $r$, positive numbers $k_{1}, \ldots, k_{r}$ such that $k_{1}<\ldots<k_{r}<k_{r+1}=\infty$ and $\left(f_{1}, \ldots, f_{r}\right) \in \mathcal{Q}_{\left(k_{2}, \ldots, k_{r+1}\right)}^{\leq-k_{1}}\left(I_{1}, \ldots, I_{r}\right)$ where $I_{j}=\left(-\frac{\pi}{2 k_{j}}, \frac{\pi}{2 k_{j}}\right)$ such that $f_{r}=f$.

By $\widehat{\Sigma}$ we denote the differential algebra of all formal power series that are multi-summable in the directions + and - , and by $\widehat{\Sigma}^{0}$ the differential algebra of multi-summable power series in the direction 0 . By $\sigma^{ \pm}: \widehat{\Sigma} \rightarrow \Sigma^{ \pm}$ we denote 'multi-summation in the direction $\pm^{ \pm}$, i.e. $\sigma^{ \pm}(\hat{f})$ is the multi-sum of $\hat{f}$ in the multi-direction with components \pm . We will write $\sigma^{ \pm}(\hat{f})=f^{ \pm}:=$ $\left(f_{1}^{ \pm}, \ldots, f_{r}^{ \pm}\right)$, or $\sigma^{ \pm}(\hat{f})=f_{r}^{ \pm}$for short.

Proposition 4.2. $\Sigma^{0}=\Sigma^{+} \cap \Sigma^{-}$and $\widehat{\Sigma}^{0}$ coincides with the set of all $\hat{f} \in \widehat{\Sigma}$ with the property that $\sigma^{+}(\hat{f})=\sigma^{-}(\hat{f}) . \sigma:=\sigma^{+}-\sigma^{-}$induces a vector space isomorphism from $\widehat{\Sigma} / \widehat{\Sigma}^{0}$ onto $\Sigma^{<0}$ with inverse

$$
\sigma^{-1}(f)=\widehat{C H}(f) \quad\left(\bmod \widehat{\Sigma}^{0}\right) .
$$

Proof. The first two statements follow immediately from Lemma 2.12 (cf. also [22, Lemma 10]). Let $\hat{f} \in \widehat{\Sigma}$. It is easily verified that, for $j=1, \ldots, r$,

$$
f_{j}^{+}-f_{j}^{-} \in \mathcal{Q}_{k_{j+1}}^{\leq-k_{1}}\left(-\frac{\pi}{2 k_{j}}, \frac{\pi}{2 k_{j}}\right)
$$

and

$$
f_{j}^{+}-f_{j}^{-} \quad\left(\bmod \mathcal{A}^{\leq-k_{j}}\right)=\left.\left(f_{j-1}^{+}-f_{j-1}^{-}\right)\right|_{\left(-\frac{\pi}{2 k_{j}}, \frac{\pi}{2 k_{j}}\right)}
$$

and thus $\sigma^{+}(\hat{f})-\sigma^{-}(\hat{f}) \in \Sigma^{<0}$. The injectivity of $\sigma$ is obvious. Now suppose $f \in \Sigma^{<0}$. By Corollary 3.5, $C H^{ \pm}(f)$ is a $\left(k_{1}, \ldots, k_{r}\right)$-sum in the direction \pm , hence $\widehat{C H}(f) \in \widehat{\Sigma} / \mathbf{C}\{z\}$ and $\sigma(\widehat{C H}(f))=C H^{+}(f)-C H^{-}(f)=f$.

The above proposition shows that the Cauchy-Heine transformation is a device by which we can construct, modulo multi-summable power series in the direction 0, a formal power series with a prescribed Stokes phenomenon 
in the direction 0 . In what follows we will consider subsets of $\Sigma^{<0}$, i.e. Stokes phenomena of a particular type.

Definition 4.3. Let $r \in \mathbf{N}$. For any $l \in\{0, \ldots, r\}$ we define the multidirection $\theta^{l \pm} \in\{+,-\}^{r}$ as follows. $\theta_{i}^{l \pm}=\mp$ for $1 \leq i \leq l$ and $\theta_{i}^{l \pm}= \pm$ for $l<i \leq r$.

Proposition 4.4. Let $\hat{f} \in \widehat{\Sigma}$. The following conditions are equivalent.

$1) . \hat{f}$ is $\left(k_{1}, \ldots ., k_{r}\right)$-summable in the multi-direction $\theta^{l \pm}$ for every $l \in$ $\{1, \ldots, r\}$.

2). For every $i \in\{1, \ldots, r\}$ and every $j \in\{i, \ldots, r\}$ there exists a quasifunction $F_{j}^{i}$ such that

$$
\begin{gathered}
F_{i}^{i} \in \mathcal{A}^{\leq-k_{i}} / \mathcal{A}^{\leq-k_{i+1}}\left(\left(-\frac{\pi}{2 k_{i}}, \frac{\pi}{2 k_{i}}\right)\right), \\
F_{j}^{i} \in \mathcal{A}^{\leq-k_{i}} / \mathcal{A}^{\leq-k_{j+1}}\left(I_{j}^{ \pm}\right) \text {if } j>i, \\
F_{j}^{i} \quad\left(\bmod \mathcal{A}^{\leq-k_{j}}\right)=\left.F_{j-1}^{i}\right|_{I_{j}^{ \pm}} \text {for all } j>i
\end{gathered}
$$

and

$$
\sigma^{\mp}(\hat{f})-\sigma^{ \pm}(\hat{f})=\sum_{i=1}^{r} F_{r}^{i} .
$$

The quasi-functions $F_{j}^{i}(j \geq i)$ are uniquely determined by $\hat{f}$. Suppose that condition 2) is satisfied and let $\left(f_{1}^{l}, \ldots, f_{r}^{l}\right)$ be the $\left(k_{1}, \ldots, k_{r}\right)$-sum of $\hat{f}$ in the multi-direction $\theta^{l \pm}$. Then we have

$$
\begin{aligned}
& f_{j}^{l}=f_{j}^{ \pm}+\sum_{i=1}^{j} F_{j}^{i} \text { if } j \leq l, \\
& f_{j}^{l}=f_{j}^{ \pm}+\sum_{i=1}^{l} F_{j}^{i} \text { if } j>l .
\end{aligned}
$$

Proof. We prove the statements of the Proposition in the case of the upper signs. Suppose that $\hat{f}$ is $\left(k_{1}, \ldots, k_{r}\right)$-summable in every multi-direction $\theta^{l+}$, with sum $\left(f_{1}^{l}, \ldots, f_{r}^{l}\right)$. Let $F_{j}^{i}:=f_{j}^{i}-f_{j}^{i-1}, j=i, \ldots, r$. We have $f_{j}^{i}=f_{j}^{i-1} \in \mathcal{A}^{\left(k_{1}\right)} / \mathcal{A}^{\leq-k_{j+1}}\left(I_{j}^{-}\right)$for all $j<i, f_{i}^{i} \in \mathcal{A}^{\left(k_{1}\right)} / \mathcal{A}^{\leq-k_{i+1}}\left(I_{i}^{-}\right)$ and $f_{i}^{i-1} \in \mathcal{A}^{\left(k_{1}\right)} / \mathcal{A}^{\leq-k_{i+1}}\left(I_{i}^{+}\right)$. For all $j>i$, both $f_{j}^{i}$ and $f_{j}^{i-1}$ belong to $\mathcal{A}^{\left(k_{1}\right)} / \mathcal{A}^{\leq-k_{j+1}}\left(I_{j}^{+}\right)$. From Lemma 2.14 we deduce that the quasi-functions $F_{j}^{i}$ have the desired properties. In order to prove the uniqueness of $F_{i}^{i}$ it 
suffices to show that $\sum_{i=1}^{r} F_{r}^{i}=0$ implies $F_{j}^{i}=0$ for all $i \in\{1, \ldots, r\}$ and all $j \geq i$. Writing

$$
F_{r}^{1}=-\sum_{i=2}^{r} F_{r}^{i}
$$

we see that

$$
\left.F_{r}^{1}\right|_{\left(-\frac{\pi}{2 k_{r}}, \frac{\pi}{2 k_{r}}\right)} \in \mathcal{A}^{\leq-k_{2}}\left(\left(-\frac{\pi}{2 k_{r}}, \frac{\pi}{2 k_{r}}\right)\right) .
$$

Hence it follows that $\left.F_{1}^{1}\right|_{\left(-\frac{\pi}{2 k_{r}}, \frac{\pi}{2 k_{r}}\right)}=0$. By Proposition 2.2 this implies that $F_{1}^{1}=0$. Using (26) and Proposition 2.3 one easily verifies that $F_{j}^{1}=0$ for all $j \geq 1$. By means of induction on $i$ it can now be shown that $F_{j}^{i}=0$ for all $i \in\{1, \ldots, r\}$ and all $j \leq i$.

Now suppose that 2) holds. Let $l \in\{1, \ldots, r\}$. Let $f_{j}^{l}:=f_{j}^{+}+\sum_{i=1}^{l} F_{j}^{i}$ if $j \in\{l+1, \ldots, r\}$ and $f_{j}^{l}:=f_{j}^{-}$for $j \in\{1, \ldots, l\}$. Obviously, $f_{j}^{l} \in$ $\mathcal{A}^{\left(k_{1}\right)} / \mathcal{A} \leq-k_{j+1}\left(I_{j}^{+}\right)$for $j>l$ and $f_{j}^{l} \in \mathcal{A}^{\left(k_{1}\right)} / \mathcal{A}^{\leq-k_{j+1}}\left(I_{j}^{-}\right)$for $j \leq l$. Furthermore, it is easily seen that $f_{j}^{l}\left(\bmod \mathcal{A}^{\leq-k_{j}}\right)=\left.f_{j-1}^{l}\right|_{I_{j}^{+}}$for all $j>l+1$, and $f_{j}^{l}\left(\bmod \mathcal{A}^{\leq-k_{j}}\right)=\left.f_{j-1}^{l}\right|_{I_{j}^{-}}$for all $j \leq l$. Noting that $f_{r}^{l}=f_{r}^{-}-\sum_{i=l+1}^{r} F_{r}^{i}$ and $F_{i}^{i}\left(\bmod \mathcal{A}^{\leq-k_{i}}\right)=0$ for all $i$ we find that, for all $j>l$,

$$
\left.f_{j}^{l}\right|_{\left(-\frac{\pi}{2 k_{r}}, \frac{\pi}{2 k_{r}}\right)}=\left.\left(f_{j}^{-}-\sum_{i=l+1}^{j} F_{j}^{i}\right)\right|_{\left(-\frac{\pi}{2 k_{r}}, \frac{\pi}{2 k_{r}}\right)} .
$$

In particular,

$$
\left.f_{l+1}^{l} \quad\left(\bmod \mathcal{A}^{\leq-k_{l+1}}\right)\right|_{\left(-\frac{\pi}{2 k_{r}}, \frac{\pi}{2 k_{r}}\right)}=\left.f_{l}^{-}\right|_{\left(-\frac{\pi}{2 k_{r}}, \frac{\pi}{2 k_{r}}\right)}
$$

and, by Proposition 2.2, this implies that $f_{l+1}^{l}\left(\bmod \mathcal{A}^{\leq-k_{l+1}}\right)=\left.f_{l}^{-}\right|_{I_{l+1}^{+}}=$ $\left.f_{l}^{l}\right|_{I_{l+1}^{+}}$. It remains to be proved that, for $j \leq l$,

$$
f_{j}^{-}=f_{j}^{+}+\sum_{i=1}^{j} F_{j}^{i} .
$$

This follows easily from $(26),(27)$ and the fact that $F_{r}^{i}\left(\bmod \mathcal{A}^{\leq-k_{j+1}}\right)=0$ for all $i>j$.

Corollary 4.5. Let $f \in \Sigma^{<0} . \widehat{C H}(f)$ is $\left(k_{1}, \ldots, k_{r}\right)$-summable in the multidirection $\theta^{l}$ such that $\theta_{i}^{l}=\mp$ for $i \leq l$ and $\theta_{i}^{l}= \pm$ for $i>l$, for every $l \in\{1, \ldots, r\}$, iff $f$ can be written in the form

$$
f=\sum_{i=1}^{r} F_{r}^{i}
$$


where the $F_{r}^{i}$ are functions with the properties mentioned in Proposition 4.4. Moreover, if these conditions are satisfied, then the multi-sum $g_{r}^{l}$ of $\widehat{C H}(f)$ in the multi-direction $\theta^{l}$ is given by

$$
g_{r}^{l}=C H^{ \pm}(f)+\sum_{i=1}^{l} F_{r}^{i} .
$$

Proof. According to Corollary 3.5 and Remark 3.6, $\widehat{C H}(f) \in \hat{\Sigma}$ and $\sigma^{ \pm}(\widehat{C H}(f))=C H^{ \pm}(f)$. Hence

$$
\sigma^{ \pm}(\widehat{C H}(f))-\sigma^{\mp}(\widehat{C H}(f))= \pm f \quad(\bmod \mathbf{C}\{z\}) .
$$

The above statements now follow immediately from Proposition 4.4.

\section{Stokes automorphisms and alien derivations.}

A multi-summable formal power series gives rise to a non-trivial Stokes phenomenon if and only if it is divergent. Stokes phenomena can be used in the analytic classification of certain local objects, e.g. locally analytic differential equations. In many cases of interest, a detailed analysis of the Stokes phenomenon associated with such an object will yield a complete set of analytic invariants of this object (i.e. quantities that are invariant under an analytic change of variable).

There is another, essentially equivalent way to obtain these invariants. Suppose, for example, that $\hat{f}=\sum_{n=1}^{\infty} a_{n} z^{n}$ is a 1 -summable power series. Then its formal Borel transform with respect to the variable $z^{-1}$ : $\sum_{n=1}^{\infty} a_{n} \frac{t^{n-1}}{\Gamma(n)}$ defines the germ at $O$ of an analytic function. This function can be continued analytically in any direction which is not a singular direction of $\hat{f}$. Similarly, with a $\left(k_{1}, \ldots, k_{r}\right)$-summable power series $\hat{f}$, one can associate $r$ such analytic functions $\varphi_{1}, \ldots, \varphi_{r} . \varphi_{1}$ is the formal Borel transform, with respect to the variable $z^{-k_{1}}$, of $\hat{f}$ and the functions $\varphi_{2}, \ldots, \varphi_{r}$ are so-called accelerates of $\varphi_{1}$. In many applications, the function $\varphi_{j}$ can be continued analytically along paths avoiding a certain discrete set of singular points whose arguments coincide with the singular directions of level $k_{j}$ of $\hat{f}$. The singularities of the functions $\varphi_{1}, \ldots, \varphi_{r}$ determine the type of Stokes phenomenon and, in an appropriate context, the analytic invariants associated with $\hat{f}$. In order to describe the nature of these singularities, J. Ecalle introduced the so-called alien derivations (cf. [8]). In this section we give an alternative definition derived directly from the Stokes phenomenon. In the sequel we restrict ourselves to the Stokes phenomenon in the direction 0 .

Example 5.1. Consider the locally analytic differential equation

$$
z^{2} \frac{d y}{d z}=f(z, y)
$$


where $f \in \mathbf{C}\{z, y\}$ such that $f(0,0)=0$ and $\frac{\partial f}{\partial y}(0,0)=\omega>0$. It possesses a unique formal solution $\hat{h} \in \mathbf{C}\{z\}_{1}$ with singular direction 0 . There exist formal power series $\hat{h}_{n} \in \mathbf{C}\{z\}_{1}, n \in \mathbf{N}$, such that

$$
\sigma^{+}(\hat{h})-\sigma^{-}(\hat{h})=\sum_{n=1}^{\infty} e^{-n \omega / z} \sigma^{-}\left(\hat{h_{n}}\right)
$$

where the series in the right-hand side converges if $\operatorname{Re}(1 / z)$ is sufficiently small (cf. [7]). $\sigma^{+}$and $\sigma^{-}$denote the multi-sums defined in the previous section (cf. Definition 4.1).

Example 5.2 (Laplace integrals). Let $\epsilon_{1}, \epsilon_{2}$ and $r$ be positive numbers, $\epsilon_{j}<2 \pi, j=1,2$ and $U:=S\left(0, \epsilon_{1}\right) \cup S\left(2 \pi-\epsilon_{2}, 2 \pi\right) \cup S((0,2 \pi), r)$ (cf. Definition 1.1). Let $\phi$ be an analytic function on $U$. Then the Laplace transform of $\phi$ will be defined as follows (cf. [7]). Let $C$ be a contour in $U$ from $\infty \exp \left(\eta_{1} i\right)$ to $\infty \exp \left\{\left(2 \pi-\eta_{2}\right) i\right\}$ where $0<\eta_{j}<\epsilon_{j}, j=1,2, \eta_{1}+\eta_{2}<\pi$. Here and in the following we apply a lemma of Ecalle which says that if a function $\psi$ is analytic on a union $\Gamma$ of finitely many unbounded contours in $\mathbf{C}$ which have asymptotes at $\infty$ then there exists an entire function $E$ such that $|\psi-E|$ is bounded on $\Gamma$ (cf. [7]; cf. also a lemma of Sato in [14]). Choosing $\Gamma=C$ and $\psi=\phi$ we see that $\int_{C} \exp (-t / z)\{\phi(t)-E(t)\} d t$ exists on $S\left(\eta_{1}-\pi / 2, \pi / 2-\eta_{2}\right)$ and it belongs to $\mathcal{A}^{<1}\left(\left(\eta_{1}-\pi / 2, \pi / 2-\eta_{2}\right)\right)$. Variation of $E$ gives a modification of this integral by elements of $\mathcal{A}^{<-1}\left(\left(\eta_{1}-\right.\right.$ $\left.\left.\pi / 2, \pi / 2-\eta_{2}\right)\right)$. Thus we get a section in $\mathcal{A}^{<1} / \mathcal{A}^{<-1}\left(\left(\eta_{1}-\pi / 2, \pi / 2-\eta_{2}\right)\right)$ which will be denoted by $\mathcal{L}_{C} \phi$. By variation of $\eta_{1}, \eta_{2}$ and $C$ we obtain a quasi-function $\mathcal{L} \phi$ in $\mathcal{A}^{<1} / \mathcal{A}^{<-1}((-\pi / 2, \pi / 2))$ which is called the Laplace integral of $\phi$.

Next suppose that there exists $\omega>0$ such that $\phi$ possesses analytic continuations $\phi^{-}$and $\phi^{+}$on $U^{-}:=U \cup(0, \omega) \cup S\left(-\epsilon_{2}, 0\right)$ and $U^{+}:=U \cup$ $\left(0, \omega e^{2 \pi i}\right) \cup S\left(2 \pi, 2 \pi+\epsilon_{1}\right)$ respectively.

Let $C^{-}$be a contour in $U^{-}$from $\infty \exp \left(-\eta_{2} i\right)$ to $\infty \exp \left\{\left(2 \pi-\eta_{2}\right) i\right\}$. Then similarly as above we may define $\mathcal{L}_{C^{-}} \phi^{-} \in \mathcal{A}^{<1} / \mathcal{A}^{<-1}\left(\left(-\pi / 2-\eta_{2}, \pi / 2-\right.\right.$ $\left.\eta_{2}\right)$ ). With $C$ as above we see that $\mathcal{L}_{C} \phi-\mathcal{L}_{C^{-}} \phi^{-}$has a representative $\int_{C_{\omega}} \exp (-t / z)\left\{\phi^{-}(t)-E^{-}(t)\right\} d t$ where $C_{\omega}=\omega+C$ and $E^{-}$is an entire function such that $\left|\phi^{-}-E^{-}\right|$is bounded on $C \cup C^{-} \cup C_{\omega}$. Hence $\mathcal{L}_{C} \phi-\mathcal{L}_{C^{-}} \phi^{-}=e^{-\omega / z} \mathcal{L}_{C}\left(\tau_{-\omega} \phi^{-}\right)$where $\tau_{-\omega}$ is the shift operator over $-\omega$ : $\left(\tau_{-\omega} \psi\right)(t):=\psi(\omega+t)$. By variation of $C^{-}$and $\eta_{2}$ we obtain an extension of $\mathcal{L}_{C^{-}} \phi^{-}$to $f^{-} \in \mathcal{A}^{<1} / \mathcal{A}^{<-1}\left(\left(-\pi / 2-\epsilon_{2}, \pi / 2\right)\right)$. Moreover, we see that

$$
\mathcal{L} \phi^{-} f^{-}=e^{-\omega / z} f_{\omega}^{-} \text {where } f_{\omega}^{-}:=\mathcal{L}\left(\tau_{-\omega} \phi^{-}\right) \in \mathcal{A}^{<1} / \mathcal{A}^{<-1}((-\pi / 2, \pi / 2)) .
$$

Similarly we define $f^{+}$starting from $\mathcal{L}_{C^{+}} \phi^{+} \in \mathcal{A}^{<1} / \mathcal{A}^{<-1}\left(\left(-\pi / 2+\eta_{1}\right.\right.$, $\left.\left.\pi / 2+\eta_{1}\right)\right)$ using a contour $C^{+}$in $U^{+}$from $\infty \exp \left(\eta_{1} i\right)$ to $\infty \exp \{(2 \pi+$ $\left.\left.\eta_{1}\right) i\right\}$. Then $f^{+} \in \mathcal{A}^{<1} / \mathcal{A}^{<-1}\left(\left(-\pi / 2, \pi / 2+\epsilon_{1}\right)\right)$ and (28) holds with the 
superscripts - replaced by + . Moreover, $f^{-}$and $f^{+}$determine a quasifunction $f$ in $\mathcal{A}^{<1} / \mathcal{A}^{\leq-1}\left(\left(-\pi / 2-\epsilon_{2}, \pi / 2+\epsilon_{1}\right)\right)$ such that $\mathcal{L} \phi\left(\bmod \mathcal{A}^{\leq-1}\right)=$ $\left.f^{ \pm}\right|_{(-\pi / 2, \pi / 2)}\left(\bmod \mathcal{A}^{\leq-1}\right)=\left.f\right|_{(-\pi / 2, \pi / 2)}$.

Thus we have a Stokes phenomenon corresponding to the crossing of the positive axis between 0 and $\omega$ :

$$
\left\{\begin{array}{l}
\mathcal{L} \phi=f^{ \pm}+e^{-\omega / z} f_{\omega}^{ \pm}, f^{+} \in \mathcal{A}^{<1} / \mathcal{A}^{<-1}\left(\left(-\pi / 2, \epsilon_{1}+\pi / 2\right)\right), \\
f^{-} \in \mathcal{A}^{<1} / \mathcal{A}^{<-1}\left(\left(-\epsilon_{2}-\pi / 2, \pi / 2\right)\right), f_{\omega}^{ \pm} \in \mathcal{A}^{<1} / \mathcal{A}^{<-1}((-\pi / 2, \pi / 2)) .
\end{array}\right\}
$$

So

(30)

$$
f^{-}=f^{+}+e^{-\omega / z} f_{\omega}, \text { where } f_{\omega}:=f_{\omega}^{+}-f_{\omega}^{-} \in \mathcal{A}^{<1} / \mathcal{A}^{<-1}((-\pi / 2, \pi / 2)),
$$

and $f_{\omega}=\mathcal{L}\left(\tau_{-\omega}\left(\phi^{+}-\phi^{-}\right)\right)$. In several applications - for example if $\phi$ is endlessly analytically continuable (cf. [7]) - it turns out that $f_{\omega}$ has similar properties as $f$, so that we may repeat the preceding procedure to get better approximations to $f^{ \pm}$. For example if $\tilde{\omega}$ is the singularity of $\phi^{+}-\phi^{-}$next to $\omega$ on $\mathbf{R}_{+}$then in (30) we have in view of (29)

$$
\begin{gathered}
e^{-\omega / z} f_{\omega}=e^{-\omega / z} F_{\omega}^{+}+e^{-\tilde{\omega} / z} f_{\tilde{\omega}}^{+} \text {where } F_{\omega}^{+} \in \mathcal{A}^{<1} / \mathcal{A}^{<-1}((-\pi / 2, \pi / 2]), \\
f_{\tilde{\omega}}^{+} \in \mathcal{A}^{<1} / \mathcal{A}^{<-1}((-\pi / 2, \pi / 2))
\end{gathered}
$$

and so

$$
e^{\tilde{\omega} / z}\left(f^{-}-f^{+}-e^{-\omega / z} F_{\omega}^{+}\right) \in \mathcal{A}^{<1} / \mathcal{A}^{<-1}((-\pi / 2, \pi / 2)) .
$$

These and other, similar, examples have motivated the following definitions.

Definition 5.3. By $\mathcal{T}^{0}$ we denote the set of formal series $f$ of the form

$$
f=\sum_{\omega \in \mathbf{C}} e^{-\omega / z} f_{\omega}
$$

where $f_{\omega} \in \mathcal{A}^{<1} / \mathcal{A}^{\leq-1}\left(\left[-\frac{\pi}{2}, \frac{\pi}{2}\right]\right)$ and $\Omega^{0}(f):=\left\{\omega \in \mathbf{C}: f_{\omega} \neq 0\right\}$ is a discrete set with the property that $\left\{|\operatorname{Im} \omega|: \omega \in \Omega^{0}(f)\right\}$ and $\left\{-\operatorname{Re} \omega: \omega \in \Omega^{0}(f)\right\}$ are bounded above. $\Omega^{0}(f)$ will be called the support of $f$.

It is easily verified that $\mathcal{T}^{0}$ is a differential algebra. For all $f, g \in \mathcal{T}^{0}$ we have $\Omega^{0}(f g) \subset \Omega^{0}(f)+\Omega^{0}(g)$ and

$$
(f g)_{\omega}=\sum_{\omega_{1}+\omega_{2}=\omega} f_{\omega_{1}} g_{\omega_{2}}
$$

and

$$
\left(\frac{d}{d z} f\right)_{\omega}=\frac{d}{d z} f_{\omega}+\omega z^{-2} f_{\omega}
$$


The unit element is the series $f$ with support $\{0\}$ and $f_{0}=1\left(\bmod \mathcal{A}^{\leq-1}\right)$. The zero element is the unique element with empty support.

Definition 5.4. By $\mathcal{S}^{1}$ we denote the set of quasi-functions $f \in$ $\mathcal{A}^{<1} / \mathcal{A}^{\leq-1}\left(\left[-\frac{\pi}{2}, \frac{\pi}{2}\right]\right)$ with the following property:

There exist a quasi-function $f^{-} \in \mathcal{A}^{<1} / \mathcal{A}^{<-1}\left(\left[-\frac{\pi}{2}, \frac{\pi}{2}\right)\right)$ such that

$$
f^{-}\left(\bmod \mathcal{A}^{\leq-1}\right)=\left.f\right|_{\left[-\frac{\pi}{2}, \frac{\pi}{2}\right)},
$$

a discrete set $\Omega^{1}(f)$ of nonnegative numbers, including 0 , and, for each $\omega \in \Omega^{1}(f)$, quasi-functions $F_{\omega} \in \mathcal{A}^{<1} / \mathcal{A}^{\leq-1}\left(\left[-\frac{\pi}{2}, \frac{\pi}{2}\right]\right)$ and $F_{\omega}^{+} \in$ $\mathcal{A}^{<1} / \mathcal{A}^{<-1}\left(\left(-\frac{\pi}{2}, \frac{\pi}{2}\right]\right)$, such that $F_{0}=f$ and, for all $\omega \in \Omega^{1}(f)$,

$$
F_{\omega}^{+} \quad\left(\bmod \mathcal{A}^{\leq-1}\right)=\left.F_{\omega}\right|_{\left(-\frac{\pi}{2}, \frac{\pi}{2}\right]}
$$

and, for each $\omega^{\prime} \in \Omega^{1}(f)-\{0\}$,

$$
e^{\omega^{\prime} / z}\left(f^{-}-\sum_{\omega \in \Omega^{1}(f): \omega<\omega^{\prime}} e^{-\omega / z} F_{\omega}^{+}\right) \in \mathcal{A}^{<1} / \mathcal{A}^{<-1}\left(\left(-\frac{\pi}{2}, \frac{\pi}{2}\right)\right) .
$$

For all $\omega \geq 0$ we define a mapping $S_{\omega}: \mathcal{S}^{1} \rightarrow \mathcal{A}^{<1} / \mathcal{A}^{\leq-1}\left(\left[-\frac{\pi}{2}, \frac{\pi}{2}\right]\right)$ by $S_{\omega}(f)=F_{\omega}$ if $\omega \in \Omega^{1}(f)$ and $S_{\omega}(f)=0$ otherwise.

By $\mathcal{T}^{1}$ we denote the set of series $f \in \mathcal{T}^{0}$ with the property that $f_{\omega} \in \mathcal{S}^{1}$ for all $\omega \in \mathbf{C}$. We define a mapping $\mathfrak{S}: \mathcal{T}^{1} \rightarrow \mathcal{T}^{0}$ by

$$
(\mathfrak{S}(f))_{\omega}=\sum_{\omega_{1}+\omega_{2}=\omega} S_{\omega_{2}}\left(f_{\omega_{1}}\right) .
$$

$\mathcal{S}^{1}$ is a differential subalgebra of $\mathcal{A}^{<1} / \mathcal{A}^{\leq-1}\left(\left[-\frac{\pi}{2}, \frac{\pi}{2}\right]\right)$ and hence it follows that $\mathcal{T}^{1}$ is a differential subalgebra of $\mathcal{T}^{0}$. For all $\omega \geq 0$ and all $f, g \in \mathcal{S}^{1}$, the following relations hold

$$
\begin{aligned}
S_{\omega}(f g) & =\sum_{\omega_{1}+\omega_{2}=\omega} S_{\omega_{1}}(f) S_{\omega_{2}}(g), \\
S_{\omega}\left(\frac{d}{d z} f\right) & =\frac{d}{d z} S_{\omega}(f)+\omega z^{-2} S_{\omega}(f) .
\end{aligned}
$$

Note that $S_{0}$ is the identity. (The mapping $S_{\omega}$ is similar to, though not identical with the mapping $S_{\omega}^{+}$defined in [12].) By means of straightforward computations it can be shown that $\mathfrak{S}$ is an injective differential algebra homomorphism. If $f, g \in \mathcal{T}^{1}$ we have

$$
(\mathfrak{S}(f g))_{\omega}=\sum_{\omega_{1}+\omega_{2}+\omega_{1}^{\prime}+\omega_{2}^{\prime}=\omega} S_{\omega_{2}}\left(f_{\omega_{1}}\right) S_{\omega_{2}^{\prime}}\left(g_{\omega_{1}^{\prime}}\right) .
$$


If $f \in \mathcal{T}^{1}$ has support $\{\omega\}$, then $\mathfrak{S}(f)=\sum_{\omega^{\prime} \geq \omega} e^{-\omega^{\prime} / z} S_{\omega^{\prime}-\omega}\left(f_{\omega}\right)$. In general,

$$
\Omega^{0}(\mathfrak{S}(f))=\bigcup_{\omega \in \Omega^{0}(f)}\left(\omega+\Omega^{1}\left(f_{\omega}\right)\right)
$$

Example 5.5. Let $f=f_{0}$ be the restriction to $\left[-\frac{\pi}{2}, \frac{\pi}{2}\right]$ of a global section of $\mathcal{A}^{(1)} / \mathcal{A}^{\leq-1}$ associated with a formal power series $\hat{f}_{0} \in \widehat{\Sigma}$ with levels $k_{1}, \ldots, k_{r}$, where $k_{1}=1$. Let $\sigma^{ \pm}\left(\hat{f}_{0}\right)=\left(f_{1}^{ \pm}, \ldots, f_{r}^{ \pm}\right)$. Suppose there exists a discrete set of positive numbers $\Omega$ and formal power series $\hat{f}_{\omega} \in \widehat{\Sigma}$, with multi-sum $\sigma^{+}\left(\hat{f}_{\omega}\right)=\left(f_{\omega, 1}^{+}, \ldots, f_{\omega, r}^{+}\right)$for each $\omega \in \Omega$, such that

$$
f_{1}^{-}-f_{1}^{+}=\sum_{\omega \in \Omega} e^{-\omega / z} f_{\omega, 1}^{+}
$$

Then $f \in \mathcal{T}^{1}, \Omega^{0}(f)=\{0\}, \Omega^{1}(f)=\Omega \cup 0$ and $\mathfrak{S}(f)_{\omega}=\left.J^{-1}\left(\hat{f}_{\omega}\right)\right|_{\left[-\frac{\pi}{2}, \frac{\pi}{2}\right]}$.

Definition 5.6. For all $n \in \mathbf{N}$ we define:

$$
\mathcal{S}^{n+1}=\left\{f \in \mathcal{S}^{1}: S_{\omega} f \in \mathcal{S}^{n} \text { for all } \omega \in \Omega^{1}(f)\right\}
$$

and

$$
\mathcal{T}^{n+1}:=\left\{f \in \mathcal{T}^{1}: \mathfrak{S}(f) \in \mathcal{T}^{n}\right\} .
$$

For all $n \in \mathbf{N}$ and $\omega=\left(\omega_{1}, \ldots, \omega_{n}\right) \in[0, \infty)^{n}$ we write: $|\omega|=\omega_{1}+\ldots+\omega_{n}$ and

$$
S_{\omega}=S_{\omega_{n}} \ldots S_{\omega_{1}}
$$

For all $f \in \mathcal{S}^{n}$ we note

$$
\Omega^{n}(f):=\left\{\omega \in[0, \infty)^{n}: S_{\omega} f \neq 0\right\} .
$$

Furthermore, we define

$$
\begin{gathered}
\mathcal{S}^{\infty}=\bigcap_{n=1}^{\infty} \mathcal{S}^{n}, \quad \mathcal{T}^{\infty}=\bigcap_{n=1}^{\infty} \mathcal{T}^{n}, \\
\mathcal{S}=\left\{f \in \mathcal{S}^{\infty}: \bigcup_{n=1}^{\infty}\left\{|\omega|: \omega \in \Omega^{n}(f)\right\} \text { is a discrete set }\right\}
\end{gathered}
$$

and

$$
\mathcal{T}=\left\{f \in \mathcal{T}^{\infty}: \bigcup_{\omega_{0} \in \Omega^{0}(f)} \bigcup_{n=1}^{\infty}\left\{\omega_{0}+|\omega|: \omega \in \Omega^{n}\left(f_{\omega_{0}}\right)\right\} \text { is discrete }\right\} .
$$

By means of induction on $n$, one easily proves the following lemma.

Lemma 5.7. Let $n \in \mathbf{N}$. $f \in \mathcal{T}^{n}$ if and only if $f_{\omega} \in \mathcal{S}^{n}$ for all $\omega \in \Omega^{0}(f)$. Similarly, $f \in \mathcal{T}$ if and only if $f_{\omega} \in \mathcal{S}$ for all $\omega \in \Omega^{0}(f)$. 
Note that, for a given $\omega$,

$$
\left((\mathfrak{S}-i d)^{n} f\right)_{\omega}=\sum_{\omega_{1}, \ldots, \omega_{n}>0} S_{\omega_{n}} \cdots S_{\omega_{1}}\left(f_{\omega-\left(\omega_{1}+\cdots+\omega_{n}\right)}\right)
$$

and this sum equals 0 for all but a finite number of integers $n$. Therefore, the infinite $\operatorname{sum} \sum_{n=0}^{\infty}(-1)^{n}(\mathfrak{S}-i d)^{n} f$, so $\mathfrak{S}^{-1} f$ is well-defined for all $f \in \mathcal{T}$ and its support is discrete. (Alternatively, we could define $\mathcal{T}$ to be the largest subalgebra of $\mathcal{T}^{1}$ that is invariant under $\mathfrak{S}$.) Thus $\mathfrak{S}$ is a differential algebra automorphism of $\mathcal{T}$. We define the 'directional alien derivation' $\Delta$ by

$$
\Delta:=\log \mathfrak{S}=\sum_{n=1}^{\infty} \frac{(-1)^{n+1}}{n}(\mathfrak{S}-i d)^{n} .
$$

$\Delta$ is a derivation of $\mathcal{T}$ (cf. [7]). We have

$$
(\Delta f)_{\omega}=\sum_{n=1}^{\infty} \frac{(-1)^{n+1}}{n} \sum_{\omega^{\prime} \in \mathbf{C} \omega^{\prime \prime} \in(0, \infty)^{n}:\left|\omega^{\prime \prime}\right|+\omega^{\prime}=\omega} S_{\omega^{\prime \prime}}\left(f_{\omega^{\prime}}\right) .
$$

$\Delta$ can be decomposed as follows: $\Delta=\sum_{\omega>0} \dot{\Delta}_{\omega}$, where $\dot{\Delta}_{\omega}$ is defined by

$$
\left(\dot{\Delta}_{\omega} f\right)_{\omega^{\prime}}=\sum_{n=1}^{\infty} \frac{(-1)^{n+1}}{n} \sum_{\omega^{\prime \prime} \in(0, \infty)^{n}:\left|\omega^{\prime \prime}\right|=\omega} S_{\omega^{\prime \prime}}\left(f_{\omega^{\prime}-\omega}\right) .
$$

$\dot{\Delta}_{\omega}$ is a derivation of $\mathcal{T}$ which transforms an element of $\mathcal{T}$ with support $\Omega$ into an element with support $\Omega+\omega$. As $\mathfrak{S}$ is a differential algebra automorphism, $\Delta$ and $\dot{\Delta}_{\omega}$ commute with $\frac{d}{d z}$. Furthermore, $\Delta_{\omega}:=e^{\omega / z} \dot{\Delta}_{\omega}$ is a derivation of $\mathcal{S}$. We have

$$
\Delta_{\omega}=\sum_{n=1}^{\infty} \frac{(-1)^{n+1}}{n} \sum_{\omega^{\prime \prime} \in(0, \infty)^{n}:\left|\omega^{\prime \prime}\right|=\omega} S_{\omega^{\prime \prime}} .
$$

For further details we refer the reader to $[\mathbf{7}, \mathbf{1 2}]$.

Definition 5.8. For all $\alpha>0, \rho_{\alpha}$ will denote the 'change of variable': $\rho_{\alpha} f(z)=f\left(z^{\alpha}\right)$. We put: $\mathfrak{S}_{\alpha}=\rho_{\alpha} \mathfrak{S} \rho_{\alpha}^{-1}, \dot{\Delta}_{\omega}^{\alpha}=\rho_{\alpha} \dot{\Delta}_{\omega} \rho_{\alpha}^{-1}$ and $\Delta_{\omega}^{\alpha}=$ $\rho_{\alpha} \Delta_{\omega} \rho_{\alpha}^{-1}$. Obviously,

$$
\dot{\Delta}_{\omega}^{\alpha}=e^{-\omega z^{-\alpha}} \Delta_{\omega}^{\alpha}
$$

Let $r \in \mathbf{N}$, let $\mathbf{k}=\left(k_{1}, \ldots, k_{r}\right)$, where $0<k_{1}<\ldots<k_{r}<k_{r+1}=\infty$ and let $\mathbf{d}=\left(d_{1}, \ldots, d_{r}\right) \in\{+,-\}^{r}$. By $\mathcal{S}_{\mathbf{k} ; \mathbf{d}}$ we denote the set of all $f \in \rho_{k_{1}} \mathcal{S}$ with the following property:

For all $n \in \mathbf{N}$ and all $\omega=\left(\omega_{1}, \ldots, \omega_{n}\right) \in \Omega^{n}\left(\rho_{k_{1}}^{-1} f\right)$, and all $j \in\{1, \ldots, r\}$, there exist quasi-functions $f_{\omega, j} \in \mathcal{Q}_{k_{j+1}}^{<k_{1}}\left(I_{j}^{d_{j}}\right)$, such that

$$
f_{\omega, j} \quad\left(\bmod \mathcal{A}^{\leq-k_{j}}\right)=\left.f_{\omega, j-1}\right|_{I_{j}^{d_{j}}}
$$


where $f_{\omega, 0}=\rho_{k_{1}} S_{\omega}\left(\rho_{k_{1}}^{-1} f\right)$.

By $\mathcal{T}_{\mathbf{k} ; \mathbf{d}}$ we denote the set of all $f \in \rho_{k_{1}} \mathcal{T}, f=\sum_{\omega \in \mathbf{C}} e^{-\omega / z^{k_{1}}} f_{\omega}$, with the property that $f_{\omega} \in \mathcal{S}_{\mathbf{k} ; \mathbf{d}}$ for all $\omega \in \mathbf{C}$. Note that $f_{\omega}=0$ unless $\omega \in$ $\Omega^{0}\left(\rho_{k_{1}}^{-1} f\right)$. We call $\Omega^{0}\left(\rho_{k_{1}}^{-1} f\right)$ the support of $f$.

It is easily verified that $\mathfrak{S}_{k_{1}} \mid \mathcal{T}_{\mathbf{k} ; \mathbf{d}}$ is again a differential algebra automorphism.

\section{Application.}

Consider the D.E. $D y=0$, where $D$ is a linear differential operator of order $n$, with coefficients in $\mathbf{C}\{z\}$. It has a formal integral of the form:

$$
\Phi(u ; z)=\Phi\left(u_{1}, \ldots, u_{n} ; z\right)=\sum_{l=1}^{n} u_{l} e^{q_{l}(z)} z^{\rho_{l}} \hat{h}_{l}
$$

where $u \in \mathbf{C}^{n}, \rho_{l} \in \mathbf{C}, q_{l} \in z^{-1 / p} \mathbf{C}\left[z^{-1 / p}\right]$, and $\hat{h}_{l} \in \mathbf{C}\left[\left[z^{1 / p}\right]\right][\log z]$ for some $p \in \mathbf{N}$. Let $k_{1}<\ldots<k_{r}$ denote the levels of $(D)$, i.e. the values of $\frac{1}{p} \operatorname{deg}\left(q_{l}-q_{m}\right)$, where $l, m \in\{1, \ldots, n\}$ such that $q_{m} \not \equiv q_{l}$. According to Theorem 1.4, $\hat{h}_{l}$ is multi-summable in every multi-direction $\mathbf{d}=$ $\left(d_{1}, \ldots, d_{r}\right) \in\{+,-\}^{r}$. Thus, for every $j \in\{1, \ldots, r\}$, there is a quasifunction $h_{l, j}^{\mathbf{d}} \in \mathcal{Q}_{k_{j+1}}^{<k_{1}}\left(I_{j}^{d_{j}}\right)$ such that $h_{l, j}^{\mathbf{d}}\left(\bmod \mathcal{A}^{\leq-k_{j}}\right)=\left.h_{l, j-1}^{\mathbf{d}}\right|_{I_{j}^{d_{j}}}$ and $h_{l, 1}^{\mathbf{d}}$ $\left(\bmod \mathcal{A}^{\leq-k_{1}}\right)=\left.J^{-1}\left(\hat{h}_{l}\right)\right|_{I_{1}^{d_{1}}}$.

Proposition 5.9. Let $r \in \mathbf{N}, 0<k_{1}<\cdots<k_{r+1}=\infty$. Let $i \in$ $\{1, \ldots, r\}$ and let $\mathbf{d}, \mathbf{d}^{\prime} \in\{+,-\}^{r}$ such that $d_{j}=d_{j}^{\prime}$ for all $j \neq i, d_{i}=$ $-d_{i}^{\prime}$. For each $j \in\{1, \ldots, r\}$, let $I_{j}:=I_{j}^{d_{j}}$ and $I_{j}^{\prime}:=I_{j}^{d_{j}^{\prime}}$. Suppose that there exist $\left(y_{1}^{1}, \ldots, y_{r}^{1}\right) \in \mathcal{Q}_{\left(k_{2}, \ldots, k_{r+1}\right)}^{<k_{1}}\left(I_{1}^{\prime}, \ldots I_{r}^{\prime}\right)$ and $\left(y_{1}^{2}, \ldots, y_{r}^{2}\right) \in$ $\mathcal{Q}_{\left(k_{2}, \ldots, k_{r+1}\right)}^{<k_{1}}\left(I_{1}, \ldots I_{r}\right)$ with the following properties:

(i) $\left(y_{1}^{2}-y_{1}^{1}\right)\left(\bmod \mathcal{A}^{\leq-k_{1}}\right)=0$

(ii) $D y_{r}^{1}=D y_{r}^{2}$.

For all $l \in\{1, \ldots, n\}$, let $q_{l}=-\omega_{l} z^{-\kappa_{l}}+\tilde{q}_{l}$, where $\tilde{q}_{l} \in \mathbf{C}\left[z^{-1 / p}\right]$, deg $\tilde{q}_{l}<$ $\operatorname{deg} q_{l}$ if $q_{l} \not \equiv 0$, otherwise $\omega_{l}=\kappa_{l}=0$. There exist $u_{l} \in \mathbf{C}$ such that, for all $j \geq i$,

$$
y_{j}^{2}-y_{j}^{1}=\sum_{l: \kappa_{l}=k_{i}, \omega_{l}>0} u_{l} e^{q_{l}(z)} z^{\rho_{l}} h_{l, j}^{\mathbf{d}} .
$$

Proof. Suppose $i<r$. According to Lemma 2.14, $y_{j}^{2}-y_{j}^{1} \in \mathcal{Q}_{k_{j+1}}^{\leq-k_{i}}\left(I_{j}\right)$ for all $j>i$, whereas $y_{i}^{2}-y_{i}^{1} \in \mathcal{Q}_{k_{i+1}}^{\leq-k_{i}}\left(\left(-\frac{\pi}{2 k_{i}}, \frac{\pi}{2 k_{i}}\right)\right)$. On the other hand, there exist complex numbers $u_{1}, \ldots, u_{n}$ such that

$$
y_{r}^{2}-y_{r}^{1}=\sum_{l=1}^{n} u_{l} e^{q_{l}(z)} z^{\rho_{l}} h_{l, r}^{\mathbf{d}} .
$$


As $y_{r}^{2}-y_{r}^{1} \in \mathcal{A}^{\leq-k_{i}}\left(I_{r}\right)$ and $e^{q_{l}}$ is of order $\kappa_{l}$, it follows that $u_{l}=0$ for all $l$ such that $\kappa_{l}<k_{i}$. From the fact that $\cos \left(\arg \omega_{l}-\kappa_{l} \arg z\right)$ changes sign on $I_{r}$ if $\kappa_{l}=k_{r}$ we infer that $u_{l}=0$ for all $l$ such that $\kappa_{l}=k_{r}$ as well. Consequently,

$$
\begin{aligned}
y_{r-1}^{2}-\left.y_{r-1}^{1}\right|_{I_{r}} & =\sum_{l: k_{i} \leq \kappa_{l}<k_{r}} u_{l} e^{q_{l}(z)} z^{\rho_{l}} h_{l, r}^{\mathrm{d}} \quad\left(\bmod \mathcal{A}^{\leq-k_{r}}\right) \\
& =\left.\sum_{l: k_{i} \leq \kappa_{l}<k_{r}} u_{l} e^{q_{l}(z)} z^{\rho_{l}} h_{l, r-1}^{\mathrm{d}}\right|_{I_{r}} .
\end{aligned}
$$

In view of the quasi-analyticity of the sheaf $\mathcal{Q}_{k_{r}}^{<k_{r}}$ (Proposition 2.2), this implies that

$$
y_{r-1}^{2}-y_{r-1}^{1}=\sum_{l: k_{i} \leq \kappa_{l}<k_{r}} u_{l} e^{q_{l}(z)} z^{\rho_{l}} h_{l, r-1}^{\mathbf{d}} .
$$

By means of induction on $r-j \in\{0, \ldots, r-i\}$ one proves that $u_{l}=0$ for all $l$ such that $\kappa_{l} \neq k_{i}$, and

$$
y_{i}^{2}-y_{i}^{1}=\sum_{l: \kappa_{l}=k_{i}} u_{l} e^{q_{l}(z)} z^{\rho_{l}} h_{l, i}^{\mathbf{d}} .
$$

Noting that $\cos \left(\arg \omega_{l}-\kappa_{l} \arg z\right)$ changes sign on $\left(-\frac{\pi}{2 k_{i}}, \frac{\pi}{2 k_{i}}\right)$ if $\kappa_{l}=k_{i}$ and $\arg \omega_{l} \notin 2 \pi \mathbf{Z}$, we conclude that $u_{l}=0$ unless $\omega_{l}>0$. The proof for the case that $i=r$ is similar.

For every $\mathbf{d} \in\{+,-\}^{r}$ and every $l \in\{1, \ldots, n\}, h_{l, r}^{\mathbf{d}}$ satisfies a linear D.E. of order $n$, with formal solutions $e^{q_{m l}} z^{\rho_{m l}} \hat{h}_{m}$, where $q_{m l}=q_{m}-q_{l}$, $\rho_{m l}=\rho_{m}-\rho_{l}, m=1, \ldots, n$. We fix a multi-direction $\mathbf{d}$ and write $h_{l, j}:=h_{l, j}^{\mathbf{d}}$ for all $j \in\{1, \ldots, r\}$. As $\hat{h}_{l}$ is multi-summable in every multi-direction $\in$ $\{+,-\}^{r}$, so in particular in every multi-direction $\in\{+,-\}^{r}$ whose first $j-1$ components coincide with those of $\mathbf{d}$, there exists, for every $j \in\{1, \ldots, r\}$, an element $h_{l, j}^{ \pm}$of $\mathcal{Q}_{k_{j+1}}^{<k_{1}}\left(I_{j}^{ \pm}\right)$with the property that

$$
h_{l, j}^{ \pm} \quad\left(\bmod \mathcal{A}^{\leq-k_{j}}\right)=\left.h_{l, j-1}\right|_{I_{j}^{ \pm}} .
$$

Let $q_{m l}=-\omega_{m l} z^{-\kappa_{m l}}+\tilde{q}_{m l}$, where $\operatorname{deg} \tilde{q}_{m l}<\operatorname{deg} q_{m l}$ if $q_{m l} \not \equiv 0$, otherwise $\omega_{m l}=\kappa_{m l}=0$. From Proposition 5.9 it follows that

$$
h_{l, j}^{-}-h_{l, j}^{+}=\sum_{m: \kappa_{m l}=k_{j}, \omega_{m l}>0} e^{-\omega_{m l} z^{-k_{j}}}\left(u_{m l} e^{\tilde{q}_{m l}(z)} z^{\rho_{m l}} h_{m, j}^{+}\right)
$$

where $u_{m l} \in \mathbf{C}$. This shows that $\left.h_{l, j-1}\right|_{\left[-\frac{\pi}{2 k_{j}}, \frac{\pi}{2 k_{j}}\right]} \in \rho_{k_{j}} \mathcal{S}^{1}$ and

$$
\left.\rho_{k_{j}} S_{\omega} \rho_{k_{j}}^{-1} h_{l, j-1}\right|_{\left[-\frac{\pi}{2 k_{j}}, \frac{\pi}{2 k_{j}}\right]}=\left.\sum_{m: \kappa_{m l}=k_{j}, \omega_{m l}=\omega} u_{m l} e^{\tilde{q}_{m l}(z)} z^{\rho_{m l}} h_{m, j-1}\right|_{\left[-\frac{\pi}{2 k_{j}}, \frac{\pi}{2 k_{j}}\right]} .
$$


Let $\mathbf{k}^{j}=\left(k_{j}, \ldots, k_{r}\right)$ and $\mathbf{d}^{j}=\left(d_{j}, \ldots, d_{r}\right)$. It is easily seen that $\left.h_{l, j-1}\right|_{\left[-\frac{\pi}{2 k_{j}}, \frac{\pi}{2 k_{j}}\right]} \in \mathcal{S}_{\mathbf{k}^{j} ; \mathbf{d}^{j}}$. It can also be considered to be an element of $\mathcal{T}_{\mathbf{k}^{j} ; \mathbf{d}^{j}}$ with support $\{0\}$. Let $\omega>0$ and define

$$
J_{l, \omega}^{(j)}:=\left\{m \in\{1, \ldots, n\}: \operatorname{deg}\left(q_{m l}+\omega z^{-k_{j}}\right)<p k_{j}\right\}
$$

(here $q_{m l}+\omega z^{-k_{j}}$ is considered a polynomial in $z^{-1 / p}$ ) and

$$
\begin{aligned}
J_{l, \omega}^{(j, s)}=\left\{\left(m_{1}, \ldots, m_{s}\right) \in\{1, \ldots, n\}^{s}: \kappa_{m_{1} l}=\cdots=\kappa_{m_{s} l}\right. & =k_{j}, \\
0 & \left.<\omega_{m_{1} l}<\cdots<\omega_{m_{s} l}<\omega\right\} .
\end{aligned}
$$

From (31), (32) and Definition 5.8 we deduce that

$$
\left.\dot{\Delta}_{\omega}^{k_{j}} h_{l, j-1}\right|_{\left[-\frac{\pi}{2 k_{j}}, \frac{\pi}{2 k_{j}}\right]}=\left.\sum_{m \in J_{l, \omega}^{(j)}} A_{l, m}(\omega) e^{q_{m l}(z)} z^{\rho_{m l}} h_{m, j-1}\right|_{\left[-\frac{\pi}{2 k_{j}}, \frac{\pi}{2 k_{j}}\right]}
$$

where

$$
A_{l, m}(\omega)=u_{m l}+\sum_{s=1}^{\infty} \frac{(-1)^{s}}{s+1} \sum_{\left(m_{1}, \ldots, m_{s}\right) \in J_{l, \omega}^{(j, s)}} u_{m_{1} l} u_{m_{2} m_{1}} \ldots u_{m m_{s}} .
$$

Defining

$$
\Phi^{(j)}(u ; z)=\sum_{l=1}^{n} u_{l} e^{q_{l}(z)} z^{\rho_{l}} h_{l, j-1} \mid\left[-\frac{\pi}{2 k_{j}}, \frac{\pi}{2 k_{j}}\right]
$$

and extending $\dot{\Delta}_{\omega}^{k_{j}}$ to $\mathcal{T}_{\mathbf{k}^{j} ; \mathbf{d}^{j}}\left[e^{q_{1}}, \ldots, e^{q_{n}}\right]$, we can summarize the above relations in one formula, for each $j \in\{1, \ldots, r\}$ ("the bridge equation of level $\left.k_{j}{ }^{\prime \prime}\right)$ :

$$
\dot{\Delta}_{\omega}^{k_{j}} \Phi^{(j)}(u ; z)=A_{\omega}^{(j)}(u) \nabla_{u} \Phi^{(j)}(u ; z)
$$

where $A_{\omega}^{(j)}(u)$ is a row vector with components

$$
A_{\omega, m}^{(j)}(u)=\sum_{l: \operatorname{deg}\left(q_{m l}+\omega z^{-k_{j}}\right)<p k_{j}} A_{l, m}(\omega) u_{l}, \quad m=1, \ldots, n .
$$

The numbers $A_{l, m}(\omega)$ depend on (the first $j-1$ components of) the multidirection $\mathbf{d}$. As $h_{l, j-1}$ is uniquely determined by $\hat{h}_{l}$ and $d_{1}, \ldots, d_{j-1}$, we can define operators $\dot{\Delta}_{\omega, d_{1}, \ldots, d_{j-1}}^{k_{j}}$ acting on formal power series and replace (34) with

$$
\dot{\Delta}_{\omega, d_{1}, \ldots, d_{j-1}}^{k_{j}} \Phi(u ; z)=A_{\omega, d_{1}, \ldots, d_{j-1}}(u) \nabla_{u} \Phi(u ; z) .
$$

The numbers $A_{l, m}(\omega)$ are analytic invariants of the differential equation. 
The bridge equation is due to J. Ecalle, cf. [8], [9]. For a different approach based on differential Galois theory we refer to a paper by J. Martinet and J.-P. Ramis [22].

Example 5.10. Let $D=D_{1} D_{2} D_{3}$ where $D_{1}=\left(z \frac{d}{d z}-1\right), D_{2}=\left(z^{2} \frac{d}{d z}-1\right)$ and $D_{3}=\left(z^{3} \frac{d}{d z}-1\right)$. All solutions of the equation $D_{1} y=0$ are multiples of the function $y(z)=z$. Therefore, $y$ is a solution of $D_{1} D_{2} y=0$ if and only if $D_{2} y=a z$ for some $a \in \mathbf{C}$. In [23] it is shown that $D_{2}$ and $D_{3}$ define isomorphisms of $\mathbf{C}\{z\}_{1}$ and $\mathbf{C}\{z\}_{2}$, respectively. Hence the equation

$$
\left(z^{2} \frac{d}{d z}-1\right) y=z
$$

has a unique formal power series solution $\hat{h}$, which is 1 -summable. The sum $h^{ \pm}$in the direction \pm can be represented by the Laplace integral

$$
h^{ \pm}(z)=\int_{l_{ \pm}} \frac{e^{-\zeta / z}}{\zeta-1} d \zeta
$$

where $l_{ \pm}$is a half line from $O$ to $\infty$ in a direction $\theta$ such that $\pm \theta \in(0,2 \pi)$. Thus all formal solutions of the homogeneous equation $\left(D_{1} D_{2}\right)$ have the form $a \hat{h}+b e^{-1 / z}$, where $a, b \in \mathbf{C}$. Let $\hat{h}_{2}$ and $\hat{h}_{3}$ denote the (unique) formal power series solutions of $D_{3}\left(e^{-1 / z} y\right)=e^{-1 / z}$, or, equivalently, of

$$
\left(z^{3} \frac{d}{d z}-1+z\right) y=1
$$

and $D_{3} y=\hat{h}$, respectively. The equation $D y=0$ has a formal integral of the form

$$
\Phi(u ; z)=u_{1} e^{-\frac{1}{2} z^{-2}}+u_{2} e^{-z^{-1}} \hat{h}_{2}(z)+u_{3} \hat{h}_{3}(z) .
$$

It has two levels: $k_{1}=1$ and $k_{2}=2$. One easily verifies that

$$
J_{3,1}^{(1)}=\{2\}, J_{2, \frac{1}{2}}^{(2)}=\{1\}, J_{3, \frac{1}{2}}^{(2)}=\{1\}
$$

and $J_{l, \omega}^{(j)}=\emptyset$ otherwise. Hence there exist complex numbers $A_{3,2}, A_{2,1}$, $A_{3,1,+}$ and $A_{3,1,-}$ such that

$$
\dot{\Delta}_{1}^{1} \Phi(u ; z)=\left(0, u_{3} A_{3,2}, 0\right) \frac{\partial}{\partial u} \Phi(u ; z)
$$

and

$$
\dot{\Delta}_{\frac{1}{2}, \pm}^{2} \Phi(u ; z)=\left(u_{2} A_{2,1}+u_{3} A_{3,1, \pm}, 0,0\right) \frac{\partial}{\partial u} \Phi(u ; z) .
$$

(Note that the 2-summability of $\hat{h}_{2}$ implies that $A_{2,1}$ is independent of $d_{1}$.) In this simple example the invariants $A_{3,2}, A_{2,1}, A_{3,1,+}$ and $A_{3,1,-}$ can be 
easily calculated. The difference between the 1 -sums $h^{+}$and $h^{-}$of $\hat{h}$ in the directions + and - is

$$
h^{-}(z)-h^{+}(z)=2 \pi i e^{-\frac{1}{z}} .
$$

This implies that $\dot{\Delta}_{1}^{1} \hat{h}=2 \pi i e^{-\frac{1}{z}}$. Hence we deduce the relation

$$
\left(z^{3} \frac{d}{d z}-1\right) \dot{\Delta}_{1}^{1} \hat{h}_{3}=\dot{\Delta}_{1}^{1}\left(z^{3} \frac{d}{d z}-1\right) \hat{h}_{3}=\dot{\Delta}_{1}^{1} \hat{h}=2 \pi i e^{-\frac{1}{z}} .
$$

It follows that $\dot{\Delta}_{1}^{1} \hat{h}_{3}=2 \pi i e^{-\frac{1}{z}} \hat{h}_{2}$ and thus $A_{3,2}=2 \pi i$.

It can be shown that the 2 -sums $h_{2}^{+}$and $h_{2}^{-}$of $\hat{h}_{2}$ in the directions + and - are given by

$$
h_{2}^{ \pm}(z)=e^{-\frac{1}{2} z^{-2}+z^{-1}} \int_{\gamma^{ \pm}(z)} e^{\frac{1}{2} \zeta^{-2}-\zeta^{-1}} \zeta^{-3} d \zeta
$$

where $\gamma^{ \pm}(z)$ is a path from $O$ to $z$, tangent at $O$ to a half line with direction $\theta$ such that $\pm \theta \in\left(\frac{\pi}{4}, \frac{3 \pi}{4}\right)$ (the expression in the right-hand side of the above formula represents the unique solution of (35) which is bounded on the half line $\arg z= \pm \frac{\pi}{2}$ ). It is easily seen that

$$
h_{2}^{-}(z)-h_{2}^{+}(z)=i \sqrt{2 \pi} e^{-\frac{1}{2} z^{-2}+z^{-1}-\frac{1}{2}}
$$

and thus $A_{2,1}=i \sqrt{\frac{2 \pi}{e}}$.

For any multi-direction $\mathbf{d} \in\{+,-\}^{2}$ let $h_{3}^{\mathbf{d}}$ denote the function defined by

$$
h_{3}^{\mathbf{d}}(z)=e^{-\frac{1}{2} z^{-2}} \int_{\gamma^{d_{2}(z)}} e^{\frac{1}{2} \zeta^{-2}} \zeta^{-3} h^{d_{1}}(\zeta) d \zeta .
$$

By means of elementary asymptotic methods (cf. [26]) it can be shown that

$$
\begin{aligned}
& h_{3}^{(+,+)} \in \mathcal{A}^{(1)}\left(\left(-\frac{\pi}{4}, \frac{5 \pi}{4}\right)\right), h_{3}^{(+,-)} \in \mathcal{A}^{(1)}\left(\left(-\frac{\pi}{2}, \frac{\pi}{4}\right)\right), \\
& h_{3}^{(-,+)} \in \mathcal{A}^{(1)}\left(\left(-\frac{\pi}{4}, \frac{\pi}{2}\right)\right), h_{3}^{(-,-)} \in \mathcal{A}^{(1)}\left(\left(-\frac{5 \pi}{4}, \frac{\pi}{4}\right)\right) .
\end{aligned}
$$

Moreover,

$$
h_{3}^{\left(d_{1},-\right)}(z)-h_{3}^{\left(d_{1},+\right)}(z)=e^{-\frac{1}{2} z^{-2}} \int_{\Gamma} e^{\frac{1}{2} \zeta^{-2}} \zeta^{-3} h^{d_{1}}(\zeta) d \zeta \in \mathcal{A}^{\leq-2}\left(\left(-\frac{\pi}{4}, \frac{\pi}{4}\right)\right)
$$

where $\Gamma$ is a closed contour tangent at $O$ to half lines with directions $\theta$ and $-\theta$, for some $\theta \in\left(\frac{\pi}{4}, \frac{3 \pi}{4}\right)$. Hence we infer that $\left\{h_{3}^{(+,-)}, h_{3}^{(+,+)}\right\}$represents an element $h_{3,1}^{+}$of $\mathcal{A}^{(1)} / \mathcal{A}^{\leq-2}\left(\left(-\frac{\pi}{2}, \frac{5 \pi}{4}\right)\right)$ and

$$
\left.h_{3,1}^{+}\right|_{\left(-\frac{\pi}{2}, \frac{\pi}{4}\right)}=h_{3}^{(+,-)} \quad\left(\bmod \mathcal{A}^{\leq-2}\right) .
$$


Similarly, one proves that $\left\{h_{3}^{(-,-)}, h_{3}^{(-,+)}\right\}$represents an element $h_{3,1}^{-}$of $\mathcal{A}^{(1)} / \mathcal{A}^{\leq-2}\left(\left(-\frac{5 \pi}{4}, \frac{\pi}{2}\right)\right)$ with the property that

$$
\left.h_{3,1}^{-}\right|_{\left(-\frac{\pi}{4}, \frac{\pi}{2}\right)}=h_{3}^{(-,+)} \quad\left(\bmod \mathcal{A}^{\leq-2}\right) .
$$

Hence it follows that $h_{3}^{\mathbf{d}}$ is the $(1,2)$-sum of $\hat{h}_{3}$ in the multi-direction $\mathbf{d}$. From (36) we deduce that

$$
A_{3,1, \pm}=\int_{\Gamma} e^{\frac{1}{2} \zeta^{-2}} \zeta^{-3} h^{ \pm}(\zeta) d \zeta
$$

Example 5.11. Let $(D)$ be a third order homogeneous linear differential equation with formal integral

$$
\Phi(u ; z)=u_{1} e^{-z^{-2}-z^{-1}} \hat{h}_{1}(z)+u_{2} e^{-z^{-1}} \hat{h}_{2}(z)+u_{3} \hat{h}_{3}(z)
$$

where $\hat{h}_{1} \equiv 1, \hat{h}_{2}=-\widehat{C H}_{2}\left(e^{-z^{-2}}\right)$ and $\hat{h}_{3}=\widehat{C H}_{1}\left(e^{-z^{-1}} C H_{2}^{+}\left(e^{-z^{-2}}\right)\right) . \hat{h}_{2}$ is 2-summable in the directions + and - with sums $h_{2}^{+}=-\mathrm{CH}_{2}^{+}\left(e^{-z^{-2}}\right)$ and $h_{2}^{-}=-\mathrm{CH}_{2}^{-}\left(e^{-z^{-2}}\right)$, respectively. Moreover, $h_{2}^{-}(z)-h_{2}^{+}(z)=e^{-z^{-2}}$. Hence it follows that $\Delta_{\omega}^{2} \hat{h}_{2}=0$ for all $\omega \neq 1$, whereas $\Delta_{1}^{2} \hat{h}_{2} \equiv 1$. Now consider $\hat{h}_{3}$. Let $h_{j, 0}=J^{-1}\left(\hat{h}_{j}\right), j=1,2,3$, and let $f:=\left.h_{3,0}\right|_{\left[-\frac{\pi}{2}, \frac{\pi}{2}\right]}$. $f$ can be represented by the restriction to $\left[-\frac{\pi}{2}, \frac{\pi}{2}\right]$ of

$$
\left\{-C H_{1}^{-}\left(e^{-z^{-1}} h_{2}^{+}\right),-C H_{1}^{+}\left(e^{-z^{-1}} h_{2}^{+}\right)\right\} .
$$

Let $f^{+}$denote the element of $\mathcal{A}^{(1)} / \mathcal{A}^{<-1}\left(\left(-\frac{\pi}{2}, \frac{\pi}{2}\right]\right)$ with representative

$$
\left\{-C H_{1}^{-}\left(e^{-z^{-1}} h_{2}^{+}\right)-e^{-z^{-1}} h_{2}^{-},-C H_{1}^{+}\left(e^{-z^{-1}} h_{2}^{+}\right)\right\}
$$

and let $f^{-}$denote the element of $\mathcal{A}^{(1)} / \mathcal{A}^{<-1}\left(\left[-\frac{\pi}{2}, \frac{\pi}{2}\right)\right)$ with representative

$$
-C H_{1}^{-}\left(e^{-z^{-1}} h_{2}^{+}\right) \text {. }
$$

Obviously, $f^{+}\left(\bmod \mathcal{A}^{\leq-1}\right)=\left.f\right|_{\left(-\frac{\pi}{2}, \frac{\pi}{2}\right]}$ and $f^{-}\left(\bmod \mathcal{A}^{\leq-1}\right)=\left.f\right|_{\left[-\frac{\pi}{2}, \frac{\pi}{2}\right)}$. It is easily seen that $f^{-}-f^{+}$is the element of $\mathcal{A}^{\leq-1} / \mathcal{A}^{<-1}\left(\left(-\frac{\pi}{2}, \frac{\pi}{2}\right)\right)$ represented by $\left\{e^{-z^{-1}} h_{2}^{-}, e^{-z^{-1}} h_{2}^{+}\right\}$. This shows that $\left.\Delta_{\omega}^{1} h_{3,0}\right|_{\left[-\frac{\pi}{2}, \frac{\pi}{2}\right]}=0$ for all $\omega \neq 1$, whereas

$$
\left.\Delta_{1}^{1} h_{3,0}\right|_{\left[-\frac{\pi}{2}, \frac{\pi}{2}\right]}=\left.h_{2,0}\right|_{\left[-\frac{\pi}{2}, \frac{\pi}{2}\right]} .
$$

Furthermore, we have

$$
\left(C H_{1}^{-}\left(e^{-z^{-1}} h_{2}^{+}\right)+e^{-z^{-1}} h_{2}^{-}\right)-C H_{1}^{+}\left(e^{-z^{-1}} h_{2}^{+}\right)=e^{-z^{-2}-z^{-1}} .
$$

Let $h_{3,1}^{-}$and $h_{3,1}^{+}$be the elements of $\mathcal{A}^{(1)} / \mathcal{A}^{\leq-2}\left(\left[-\frac{\pi}{2}, \frac{\pi}{2}\right)\right)$ and $\mathcal{A}^{(1)} / \mathcal{A}^{\leq-2}\left(\left(-\frac{\pi}{2}\right.\right.$, $\left.\left.\frac{\pi}{2}\right]\right)$, represented by $-C H_{1}^{-}\left(e^{-z^{-1}} h_{2}^{+}\right)$and $\left\{-C H_{1}^{-}\left(e^{-z^{-1}} h_{2}^{+}\right)-e^{-z^{-1}} h_{2}^{-}\right.$, $\left.-\mathrm{CH}_{1}^{+}\left(e^{-z^{-1}} h_{2}^{+}\right)\right\}$, respectively. Now, $\left(h_{3,1}^{-},-C H_{1}^{-}\left(e^{-z^{-1}} h_{2}^{+}\right)\right)$is the $(1,2)$ sum of $\hat{h}_{3}$ in the direction $(-, 0),\left(h_{3,1}^{+},-C H_{1}^{-}\left(e^{-z^{-1}} h_{2}^{+}\right)-e^{-z^{-1}} h_{2}^{-}\right)$is 
the $(1,2)$-sum of $\hat{h}_{3}$ in the direction $(+,-)$ and $\left(h_{3,1}^{+},-C H_{1}^{+}\left(e^{-z^{-1}} h_{2}^{+}\right)\right)$is the $(1,2)$-sum of $\hat{h}_{3}$ in the direction $(+,+)$. From $(37)$ we deduce that $\left.\Delta_{\omega}^{2} h_{3,1}^{-}\right|_{\left[-\frac{\pi}{4}, \frac{\pi}{4}\right]}=0$ for all $\omega>0$, whereas $\left.\Delta_{\omega}^{2} h_{3,1}^{+}\right|_{\left[-\frac{\pi}{4}, \frac{\pi}{4}\right]}=0$ for all $\omega \neq 1$ and $\left.\Delta_{1}^{2} h_{3,1}^{+}\right|_{\left[-\frac{\pi}{4}, \frac{\pi}{4}\right]}=-e^{-z^{-1}}$.

Combining the above results and applying them to the formal integral we find

$$
\begin{aligned}
\dot{\Delta}_{1}^{1} \Phi(u ; z) & =\left(0, u_{3}, 0\right) \frac{\partial}{\partial u} \Phi(u ; z), \\
\dot{\Delta}_{1,-}^{2} \Phi(u ; z) & =\left(u_{2}, 0,0\right) \frac{\partial}{\partial u} \Phi(u ; z)
\end{aligned}
$$

and

$$
\dot{\Delta}_{1,+}^{2} \Phi(u ; z)=\left(u_{2}-u_{3}, 0,0\right) \frac{\partial}{\partial u} \Phi(u ; z)
$$

\section{References}

[1] W. Balser, From divergent power series to analytic functions, Lecture Notes in Math., 1582 (1994), Springer, Berlin.

[2] W. Balser, B.L.J. Braaksma, J.-P. Ramis and Y. Sibuya, Multisummability of formal power series solutions of linear ordinary differential equations, Asymptotic Analysis, 5 (1991), 27-45.

[3] G.D. Birkhoff, Singular points of ordinary linear differential equations, Trans. Amer. Math. Soc., 10 (1909), 436-470.

[4] R.P. Boas, Entire functions, Academic Press Inc., New York, 1954.

[5] B.L.J. Braaksma, Multisummability and Stokes multipliers of linear meromorphic differential equations, J. Diff. Eq., 92 (1991), 45-75.

[6] _ Multisummability and Stokes phenomenon for linear meromorphic differential equations, in 'Trends and Developments in Ordinary Differential Equations', Proc. International Symposium, Kalamazoo, 1993 (Yousef Alavi and Po-Fang Hsieh, eds.), (1994), 15-26, World Scientific, Singapore.

[7] B. Candelpergher, J.C. Nosmas and F. Pham, Approche de la résurgence, Actualités Math., Hermann, Paris, 1993.

[8] J. Ecalle, Les fonctions résurgentes, III, Publ. Mathématiques d'Orsay, 1985.

[9] _ The acceleration operators and their applications to differential equations, quasianalytic functions, and the constructive proof of Dulac's conjecture, Proc. International Congress of Mathematicians (Ichiro Satake, ed.), Kyoto 1990, Vol. 2, 1249-1258, Math. Soc. Japan, Springer, Tokyo, Berlin, 1991.

[10] L. Hörmander, Notions of convexity, Birkhäuser, Basel, 1994.

[11] Yu.S. Il'yashenko, Finiteness theorems for limit cycles, Translations of Mathematical Monographs, Vol. 94, AMS, 1991.

[12] G.K. Immink, Multisummability and the Stokes phenomenon, J. Dyn. Contr. Syst., 1 (1995), 483-534. 
[13] W.B. Jurkat, Meromorphe differentialgleichungen, Lecture Notes in Math., 637 (1978), Springer, Berlin.

[14] H. Komatsu, Operational calculus, hyperfunctions and ultradistributions, Algebraic Analysis, 1 (1988), 357-372.

[15] V. Kostov, The Stokes' multipliers and the Galois' group of a non-Fuchsian system and the generalised Phragmen-Lindelöff principle, Funkc. Ekv., 36(2) (1983), 329-357.

[16] B.Ja. Levin, Distribution of zeros of entire functions, Transl. of Math. Monographs.

[17] M. Loday-Richaud, Stokes phenomenon, multisummability and differential Galois groups, Ann. Inst. Fourier, 44 (1994), 849-906.

[18] B. Malgrange, Remarques sur les équations différentielles à points singuliers irréguliers, Lecture Notes in Math., 712 (1979), 77-86, Springer, Berlin.

[19] __ Equations différentielles à coefficients polynomiaux, Progress in Mathematics, Birkhäuser, Basel, 1991.

[20] _ Sommation des séries divergentes, Expo. Math., 13 (1995), 163-222.

[21] B. Malgrange and J.-P. Ramis, Fonctions multisommables, Ann. Inst. Fourier, 42 (1992), 353-368.

[22] J. Martinet and J.-P. Ramis, Elementary acceleration and multisummability, Ann. Inst. H. Poincaré, Physique Théorique, 54(1) (1991), 1-71.

[23] J.-P. Ramis, Les séries k-sommables et leurs applications, in 'Analysis, Microlocal Calculus and Relativistic Quantum Theory', Proceedings "Les Houches", 1979, Springer Lecture Notes in Physics 126, Springer, Berlin, (1980), 178-199.

[24] Séries divergentes et théories asymptotiques, Panoramas et synthèses, Soc. Math. France, Paris, 121 (1993).

[25] J.-P. Ramis and Y. Sibuya, A theorem concerning multisummability of formal solutions of nonlinear meromorphic differential equations, Ann. Inst. Fourier, 42 (1992).

[26] W. Wasow, Asymptotic expansions for ordinary differential equations, Interscience Publishers, New York, 1965.

Received May 20, 1997 and revised January 22, 1998.

University of Groningen

P.O. BOX 800

9700 AV Groningen

The Netherlands

E-mail address: braaksma@math.rug.nl

University of Groningen

P.O. BOX 800

9700 AV GRoningen

The Netherlands

E-mail address: immink@eco.rug.nl

University of Minnesota

MinneAPOLIS MN 55455

E-mail address: sibuya@math.umn.edu 